\title{
Viscous Taylor droplets in axisymmetric and planar tubes: from Bretherton's theory to empirical models
}

\author{
Gioele Balestra · Lailai Zhu · François Gallaire
}

Received: date / Accepted: date

\begin{abstract}
The aim of this study is to derive accurate models for quantities characterizing the dynamics of droplets of non-vanishing viscosity in capillaries. In particular, we propose models for the uniform-film thickness separating the droplet from the tube walls, for the droplet front and rear curvatures and pressure jumps, and for the droplet velocity in a range of capillary numbers, $\mathrm{Ca}$, from $10^{-4}$ to 1 and inner-to-outer viscosity ratios, $\lambda$, from 0 , i.e. a bubble, to high viscosity droplets. Theoretical asymptotic results obtained in the limit of small capillary number are combined with accurate numerical simulations at larger $\mathrm{Ca}$. With these models at hand, we can compute the pressure drop induced by the droplet. The film thickness at low capillary numbers $\left(\mathrm{Ca}<10^{-3}\right)$ agrees well with Bretherton's scaling for bubbles as long as $\lambda<1$. For larger viscosity ratios, the film thickness increases monotonically, before saturating for $\lambda>10^{3}$ to a value $2^{2 / 3}$ times larger than the film thickness of a bubble. At larger capillary numbers, the film thickness follows the rational function proposed by Aussillous \& Quéré $[5]$ for bubbles, with a fitting coefficient which
\end{abstract}

\section{Gioele Balestra}

Laboratory of Fluid Mechanics and Instabilities, EPFL, CH1015 Lausanne, Switzerland

Tel.: +41216935312

Fax: +41216937855

E-mail: gioele.balestra@epfl.ch

Lailai Zhu

Laboratory of Fluid Mechanics and Instabilities, EPFL, CH1015 Lausanne, Switzerland

Linné Flow Centre and Swedish e-Science Research Centre (SeRC), KTH Mechanics, SE 10044 Stockholm, Sweden

Department of Mechanical and Aerospace Engineering, Princeton University, NJ 08544, USA

François Gallaire

Laboratory of Fluid Mechanics and Instabilities, EPFL, CH1015 Lausanne, Switzerland is viscosity-ratio dependent. This coefficient modifies the value to which the film thickness saturates at large capillary numbers. The velocity of the droplet is found to be strongly dependent on the capillary number and viscosity ratio. We also show that the normal viscous stresses at the front and rear caps of the droplets cannot be neglected when calculating the pressure drop for $C a>10^{-3}$.

Keywords Film thickness - Droplet velocity · Pressure drop $\cdot$ Lubrication theory $\cdot$ Numerical simulations

\section{List of symbols}

A coefficient for flow profile

$B$ coefficient for flow profile

$C \quad$ coefficient for interface profile of static meniscus

$\mathscr{C}$ mean curvature of droplet interface

$D \quad$ coefficient for interface profile of static meniscus

$c_{1}, c_{2} \quad$ coefficient for fitting law of $P, \bar{P}$

$\mathrm{Ca}$ capillary number based on droplet velocity

$C a_{\infty} \quad$ capillary number based on mean outer velocity

$F \quad$ coefficient for minimum film thickness

$\bar{F} \quad$ averaged $F$ coefficient

$G \quad$ coefficient for minimum film thickness

$H \quad$ thickness of film between wall and droplet

$H_{\text {min }} \quad$ minimum film thickness

$H_{\infty} \quad$ uniform film thickness

$H_{\infty}^{\star} \quad$ critical uniform film thickness for recirculations

$K \quad$ coefficient for linearized lubrication equation

I identity tensor

$L_{d} \quad$ droplet length

$M \quad$ coefficient for pressure model

$m \quad$ rescaled viscosity ratio

$N \quad$ coefficient for pressure model

n unit vector normal to the droplet interface

$O \quad$ coefficient for pressure model 
$P \quad$ coefficient for interface profile of static meniscus

$\bar{P} \quad$ averaged $P$ coefficient

$p \quad$ pressure

$p_{\text {linear }}$ pressure if constant gradient

$Q \quad$ coefficient for uniform film thickness model

$q \quad$ volume flux

$R \quad$ capillary tube radius or half width

Re Reynolds number

$r \quad$ radial direction (axisymmetric geometry)

$\tilde{r} \quad$ half width of droplet

$S \quad$ coefficient for classical pressure model

$t \quad$ time

$T \quad$ coefficient for plane curvature model

$U_{d} \quad$ droplet velocity

$U_{\infty} \quad$ average outer flow velocity

$u_{\infty} \quad$ outer far-field velocity profile

u velocity field

$u \quad$ streamwise velocity

$v \quad$ spanwise velocity

$x \quad$ streamwise direction (planar geometry)

$y \quad$ spanwise direction (planar geometry)

$z \quad$ axial direction (axisymmetric geometry)

$Z \quad$ coefficient for plane curvature model

\section{Greek symbols}

$\alpha \quad$ parameter for solution of linear lubrication equation

$\beta \quad$ coefficient for plane curvature model

$\Delta \quad$ difference between inner and outer quantities

$\Delta p^{\mathrm{NP}} \quad$ pressure correction due to non-parallel flow effects

$\Delta p_{\text {tot }} \quad$ total pressure drop

$\gamma \quad$ surface tension

$\eta \quad$ rescaled film thickness

$\kappa \quad$ plane curvature of droplet interface in $(z, r)$ or $(x, y)$

$\kappa_{f, r} \quad$ plane curvature at the front/rear droplet extremities

$\lambda$ inner-to-outer dynamic viscosity ratio

$\mu \quad$ dynamic viscosity

$\xi \quad$ rescaled axial direction

$\sigma \quad$ total stress tensor

$\tau \quad$ viscous stress tensor

$\phi \quad$ phase of solution of linear lubrication equation

$\chi \quad$ geometric coefficient

$\Omega \quad$ droplet volume or area

\section{Subscripts and superscripts}

$\begin{array}{ll}f & \text { front cap } \\ i & \text { inner } \\ o & \text { outer } \\ r & \text { rear cap } \\ z z & \text { normal tensor component in the axial direction }\end{array}$

\author{
Abbreviations \\ 2D \\ two-dimensional \\ 3D three-dimensional \\ ALE arbitrary Lagrangian-Eulerian \\ BIM boundary integral method \\ FEM finite element method
}

\section{Introduction}

Two-phase flows in microfluidic devices gained considerably in importance during the last two decades [55,21]. The key for success of these microfluidic tools is the fluid compartmentalization, allowing the miniaturization and manipulation of small liquid portions at high throughput rates with a limited number of necessary controls. Reduced liquid quantities are commonly used as individual reactors in several biological and chemical applications [34], as well as in industrial processes [1] and in micro-scale heat and mass transfer equipments [40,58,44]. Bubbles and droplets often flow in microchannels with a round or rectangular/square crosssection [35, 32, 44].

The dynamics of a bubble in a microchannel has been the subjects of several studies, since the seminal works of Fairbrother \& Stubbs [15], Taylor [56] and Bretherton [9]. These long bubbles, also referred to as Taylor bubbles, flowing in a tube of radius $R$, have been characterized by the thickness $H_{\infty}$ of the uniform film separating them from the tube walls, the minimum thickness $H_{\min }$ of the film, the plane curvature of the front and rear caps in the $(z, r)$ or $(x, y)$ plane, $\kappa_{f}$ and $\kappa_{r}$, as well as by their velocity $U_{d}$. Bretherton [9] used a lubrication approach to derive the asymptotic scalings in the limit of small capillary numbers, $C a=\mu_{o} U_{d} / \gamma$, where $\mu_{o}$ is the dynamic viscosity of the outer fluid and $\gamma$ the surface tension. In particular, Bretherton [9] showed that in the limit of $\mathrm{Ca} \rightarrow 0$ the film thickness scales as $H_{\infty} / R \sim 0.643(3 \mathrm{Ca})^{2 / 3}$ and that the plane curvature of the front and rear caps scales as $\kappa_{f, r} R \sim 1+\beta_{f, r}(3 C a)^{2 / 3}$, with $\beta_{f, r}$ a different coefficient for front and rear caps. The uniform thin-film region is connected to the static cap of constant curvature at the extremities of the bubble through a dynamic meniscus [10] (see Fig. 1). The counterpart theory for a bubble in a square duct was derived by Wong et al. [59,60]. However, these scalings agree with Taylor's experimental results [56] only in the small $C a$ limit, namely when $C a \lesssim 10^{-3}$. In order to understand the dynamics of confined bubbles in a broader parameter range, researchers have performed experiments $[11,5,16,24,7]$ as well as numerical simulations [53,48, 47, 19, 20, 26, 35, 36, 22, 3,2, 39]. As an outcome, several correlations have been proposed for the evolutions of the relevant quantities as a function of the different parameters (see for example Ref. [24] and Ref. [39]). Among them, Aussillous 


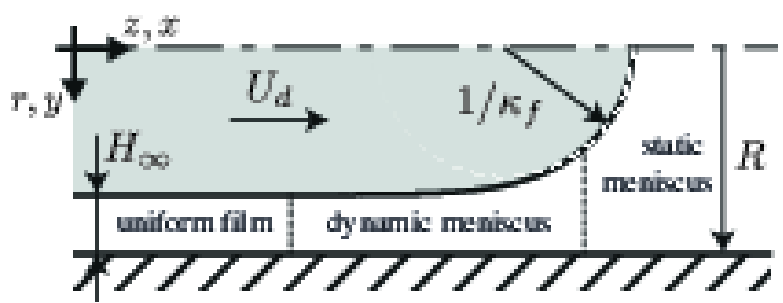

Fig. 1 Sketch of the front meniscus of the bubble advancing at velocity $U_{d}$ in a capillary of radius $R$ with indication of the uniform thin-film region of thickness $H_{\infty}$, the dynamic meniscus region and the static meniscus region. The plane curvature $\kappa_{f}$ of the front static cap in the $(z, r)$ or $(x, y)$ plane is also highlighted.

\& Quéré [5] proposed an ad-hoc rational function with a fitting parameter for the film thickness which is in good agreement with the experimental results of Taylor [56] for capillary numbers up to 1 . The two recent works of Klaseboer et al. [33] and Cherukumudi et al. [12] tried to put a theoretical basis to this extended Bretherton's theory for larger $\mathrm{Ca}$.

In contrast to bubbles, which have experienced a vast interest of the scientific community, little amount of effort has been made for droplets whose viscosities are comparable to or much larger than that of the outer fluid. Yet, droplets of arbitrary viscosities are crucial for Lab-on-a-Chip applications [4]. A first theoretical investigation of the effect of the inner phase viscosity was conducted by Schwartz et al. [52], motivated by the discrepancy in the predicted and the measured film thicknesses of long bubbles in capillaries. They demonstrated that the non-vanishing inner-to-outer viscosity ratio could thicken the film. Hodges et al. [28] further extended the theory and showed that the film becomes even thicker at intermediate viscosity ratios. Numerical simulations have been performed to investigate the droplets in capillaries 443 , 57,36].

Models predicting the characteristic quantities such as the uniform film thickness and the meniscus curvatures of droplets in capillaries over a wide range of capillary numbers are still missing. For example, the velocity of a droplet of finite viscosity flowing in a channel still remains a simple question yet an open challenge. Such a prediction is, however, of paramount importance for the correct design of droplet microfluidic devices. As an example, Jakiela et al. [30] performed extensive experiments for droplets in square ducts, showing complex dependencies of the droplet velocity on the capillary number, viscosity ratio and droplet length. Also, what is the pressure drop induced by the presence of a drop in a channel? This question is crucial and has been the subjects of recent works, for example Refs. [58, 37]. Other quantities, such as the minimum film thickness $H_{\mathrm{min}}$, have to be accurately predicted as well. $H_{\min }$ becomes essential for heat transfer or cleaning of microchannels applications [42,31]. Furthermore, being able to predict the flow field in- side and outside of the droplet is crucial if one is interested in the mixing capabilities of the system.

Here, we aim at bridging this gap by combining asymptotic derivations with accurate numerical simulations to propose physically inspired empirical models, for the characteristic quantities of a droplet of arbitrary viscosity ratio flowing in an axisymmetric or planar capillary with a constant velocity. The model coefficients are specified by fitting laws. The present work provides the reader with a rigorous theoretical basis, which can be exploited to understand the dynamics of viscous droplets. The considered capillary numbers vary from $10^{-4}$ to 1 and the inner-to-outer viscosity ratio from 0 to $10^{5}$. Following the work of Schwartz et al. [52], we extend the low-capillary-number asymptotical results obtained with the lubrication approach of Bretherton [9] for bubbles to viscous droplets. Numerical simulations based on finite element method (FEM) employing the arbitrary Lagrangian-Eulerian (ALE) formulation are performed to validate the theoretical models and then extend them to the large-capillary-number range, $C a \sim O(1)$, where the lubrication analysis fails.

The paper is structured in a way to build, step by step, the models for the uniform film thickness, the front and rear droplet's interface plane curvatures as well as those for the front and rear pressure drops required to compute the total pressure drop along a droplet in a channel. We present the problem setup, governing equations, numerical methods and the validations in Sec. 2. The flow fields inside and outside of the droplets as a function of the capillary numbers and viscosity ratios are shown in Sec. 3 . In particular, the flow profiles in the uniform-film region are derived in Sec. 3.1 and the flow patterns are presented in Sec. 3.2 The theoretical part starts with the asymptotic derivation of the model for the uniform film thickness in Sec.4. The derivation of the lubrication equation is detailed in Sec. 4.1, followed by the film thickness model in Sec. 4.2 and its extension to larger capillary numbers in Sec. 4.3. With the knowledge of the film thickness, the droplet velocity can be computed analytically (see Sec. 5). The minimum film thickness separating the droplet form the channel walls is discussed in Sec.6 To build a total pressure drop model, one still needs the knowledge of the front and rear caps mean curvatures (see Sec. 7.1), the front and rear pressure jumps (see Sec. 7.2 and 7.4) and the front and rear normal viscous stress jumps (see Sec. 7.3. The stresses evolutions at the channel centerline and at the wall are presented in Sec. 8.1 and Sec. 8.2, respectively. Eventually, one can sum up all these contributions to build the total pressure drop, which is described in Sec. 8.3 We summarize our results in Sec. 9 . 


\section{Governing equations and numerical methods}

\subsection{Problem setup}

We consider an immiscible droplet of volume $\Omega$ and dynamic viscosity $\mu_{i}$ translating at a steady velocity $U_{d}$ in a planar channel/axisymmetric tube of width/diameter $2 R$ filled with an outer fluid of dynamic viscosity $\mu_{o}$. The volume flux of the outer fluid is $q_{o}$, resulting in an average velocity $U_{\infty}=q_{o} /(2 R)$ and $U_{\infty}=q_{o} /\left(\pi R^{2}\right)$ for the planar and axisymmetric configuration, respectively (see Fig. 2). Given the small droplet velocity and size, the Reynolds number $R e$ is small and inertial effects can be neglected. Buoyancy is also neglected. The relevant dimensionless numbers include the droplet capillary number $C a=\mu_{o} U_{d} / \gamma$ with $\gamma$ being the surface tension of the droplet interface and the dynamic viscosity ratio $\lambda=\mu_{i} / \mu_{o}$ between the droplet and the outer fluid. The capillary number based on the mean flow velocity is $C a_{\infty}=\mu_{o} U_{\infty} / \gamma$. For the numerical simulations, the droplet

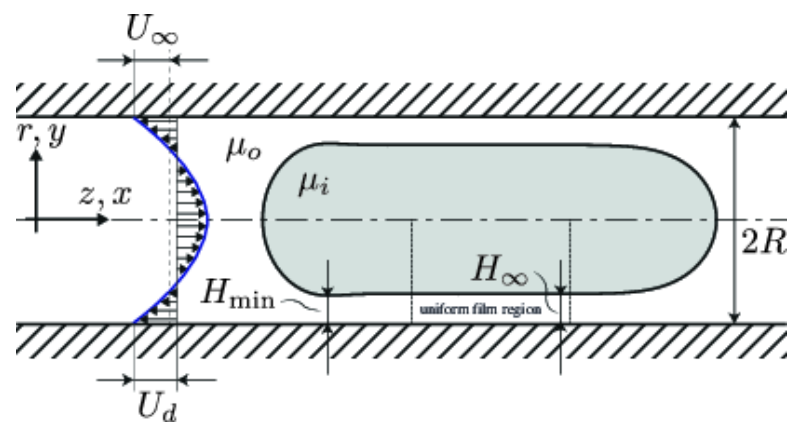

Fig. 2 Sketch of the axisymmetric $(z, r)$ and planar $(x, y)$ configurations in the frame of reference moving with the droplet. The flow profiles in the uniform film region are shown in Fig. 7

capillary number has been varied within $10^{-4} \lesssim C a \lesssim 1$ to guarantee that the lubrication film is only influenced by the hydrodynamic forces and the dynamics is steady. For smaller capillary numbers, non-hydrodynamic forces such as disjoining pressures due to intermolecular forces might come into play as reported by the recent experiments [29]; while for larger capillary numbers, instability and unsteadiness might arise, where the droplet might form a re-entrant cavity at its rear [57], which eventually breaks up into satellite droplets The viscosity ratios investigated numerically are from the well-known bubble limit of $\lambda=0$ [9] to highlyviscous droplets of $\lambda=100$ that has been scarcely investigated.

We consider both a three-dimensional axisymmetric tube, and a two-dimensional planar channel featured by the spanwise invariance. Note that the latter configuration does not correspond to the Hele-Shaw-cell-like microfluidic chips, where Darcy or Brinkman equations are more appropriate to describe the flow [8,45].
It is worth noting that the confined droplet has to be long enough to develop a region of uniformly thick film at its center [10] (see Fig. 2). However, a long axisymmetric droplet is likely to become unstable to the Rayleigh-Plateau instability. The uniform film region would resemble to a coaxial jet, which is known to be unstable to perturbations with a wavelength longer than $2 \pi\left(R-H_{\infty}\right)$ [14]. Within this range of droplet lengths, we found that the effect of droplet volume $\Omega$ is insignificant and hence it is fixed to $\Omega / R^{3}=12.9$ for the axisymmetric geometry and $\Omega / R^{2}=9.3$ for the planar case.

\subsection{Governing equations}

The governing equations are the incompressible Stokes equations for the velocity $\mathbf{u}=(u, v)$ and pressure $p$ :

$$
\begin{aligned}
\nabla \cdot \mathbf{u} & =0 \\
\mathbf{0} & =\nabla \cdot \sigma,
\end{aligned}
$$

where $\sigma=-p \mathbf{l}+\mu\left[(\nabla \mathbf{u})+(\nabla \mathbf{u})^{T}\right]$ denotes the total stress tensor and $\mu$ the dynamic viscosity as $\mu_{i}$ (resp. $\mu_{o}$ ) inside (resp. outside) the droplet.

The imposed dynamic boundary conditions at the interface are the continuity of tangential stresses

$\Delta[(\mathbf{I}-\mathbf{n n}) \cdot(\boldsymbol{\sigma} \cdot \mathbf{n})]=\mathbf{0}$,

and the discontinuity of normal stresses due to the Laplace pressure jump

$\Delta(\sigma \cdot \mathbf{n})=-\gamma \mathscr{C} \mathbf{n}$.

$\Delta$ denotes the difference between the inner and outer quantities, $\mathbf{n}$ the unit normal vector on the interface towards the outer fluid, and $\mathscr{C}=\nabla_{S} \cdot \mathbf{n}$ the interfacial mean curvature ( $\nabla_{S}$ is the surface gradient). The plane curvature of interface in the $(z, r)$ or $(x, y)$ plane is denoted as $\kappa$ and its value at the front and rear droplet extremities is given by $\kappa_{f}$ and $\kappa_{r}$, respectively. The mean curvature at the front and rear droplet extremities, which lie on the symmetry axis, is therefore given by $\mathscr{C}_{f, r}=\chi \kappa_{f, r}$, where $\chi=1$ or 2 for a planar or axisymmetric configuration, respectively. At any other point on the droplet interface, the mean curvature is given by the sum of the two principal curvatures.

\subsection{Numerical methods and implementations}

Equations (1)-(2) with boundary conditions (3)-(4) are solved by the commercial FEM package COMSOL Multiphysics and the interface is well represented by the arbitrary LagrangianEulerian (ALE) technique. Compared to the commonly known 
diffuse interface methods such as volume-of-fluid, phasefield, level-set and front-tracking all relying on a fixed Eulerian grid, the ALE approach captures the interface more accurately. Since the interface is always explicitly represented by the discretization points (see Fig. 3), the fluid quantities (viscosity, density, etc.), pressure and normal viscous stresses, are discontinuous across the interface. This technique has been used to simulate three-dimensional bubbles in complex microchannels [3], liquid films coating the interior of cylinders [25], two-phase flows with surfactants [17, [18] and head-on binary droplet collisions [41], to name a few. The Moving Mesh module of COMSOL Multiphysics has been recently employed in Refs. [49,23] to investigate the inertial and capillary migration of bubbles in round and rectangular microchannels.

Despite the high fidelity in interface capturing, it is commonly more challenging to develop in-house ALE implementations compared to the diffuse interface counterparts. Additional difficulty arises in the case of large interfacial deformations, when the computational mesh might become highly nonuniform and skewed. It is therefore necessary to remesh the computational domain and to obtain all the quantities on the new mesh via interpolation. Hence, special expertise in scientific computing and tremendous amount of development effort is required to implement an in-house ALEbased multi-phase flow solvers, which have prevented large portion of the research community from enjoying the high fidelity and elegance of the ALE methods.

Hereby, large mesh deformations can be avoided not only thanks to the convenient moving mesh module of COMSOL, but also by solving the problem in the moving frame of droplet. To achieve so, we impose a laminar Poiseuille inflow of mean velocity $U_{\infty}-U_{d}$ at the inlet of the channel and the velocity $-U_{d}$ at the walls, where the unknown droplet velocity $U_{d}$ is obtained as part of the solution together with that of the flow field, at each time step, by applying an extra constraint of zero volume-integrated velocity inside the droplet. Such constraints with additional unknowns are imposed in COMSOL Multiphysics by utilizing its so-called 'Global Equations'. This strategy ensures that the deforming droplet barely translates in the streamwise direction, staying approximately at its initial position (say in the center of the domain). Hence, the mesh quality and the robustness of the ALE formulation is appropriately guaranteed.

In this work, we are only concerned with the steady dynamics of the droplet reaching its equilibrium shape. We do not solve the steady Stokes Eq. (2) strictly but maintain a negligible time-derivative term $R e \frac{\partial \mathbf{u}}{\partial t}$ for time marching. This procedure can be seen as an iterative scheme to find steady solutions of the Stokes equations. Since, indeed, the time-derivative term vanishes when the equilibrium state is reached, the solutions to the steady Stokes equations are eventually obtained.
It has to be stressed the computed transient dynamics is not physical and only the final steady solution should be considered. Theoretically, the stability of the latter might be affected by the time-derivative term. However, in practice, no unstable phenomena or multiple-branch solutions were observed in our study. We monitored, during each simulation, the temporal evolution of the velocity, the mean and minimum film thickness as well as the front and rear plane curvatures of the droplet, which exhibited precise time-invariance without exception when the equilibrium state was reached. Our method therefore converges to valid stationary solutions, which will be seen in Sec. 2.4 to compare well with asymptotic estimates and numerical solutions from the literature.

To reduce the computational cost, half of the channel is considered and axisymmetric or symmetric boundary conditions are imposed at the channel centerline for the axisymmetric and planar configurations, respectively. The setup in COMSOL Multiphysics is intrinsically parallel and the computing time required for an individual case needs no more than one hour based on a standard desktop computer.

A typical mesh is shown in Fig. 3 Triangular/quadrilateral elements are used to discretize the domain inside/outside the droplet. Furthermore, a mesh refinement is performed to best resolve the thin lubrication film (see inset of Fig. 3). It is worth-noting that quadrilateral elements have to be used to discretize the thin film because this region might undergo large radial deformation resulting in highly distorted and skewed triangular elements if used.

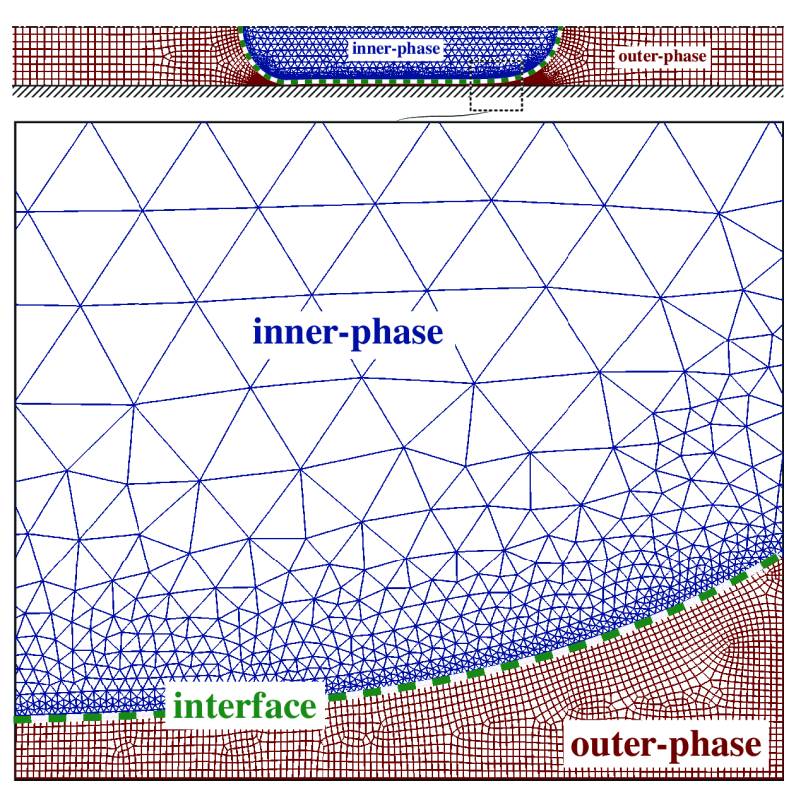

Fig. 3 Computational mesh. Inset: mesh refinement in the thin-film region. The triangular inner-phase (blue) and quadrilateral outer-phase meshes (red) are separated by the explicitly discretized interface (dashed green). 


\subsection{Validation}

Our numerical results are first validated for a bubble $(\lambda=0)$ comparing the film thickness with the classical asymptotic theory $H_{\infty} / R \sim 0.643(3 \mathrm{Ca})^{2 / 3}$ of Bretherton in the low- $\mathrm{Ca}$ limit [9] (see Fig. 4). Excellent agreement is revealed even when the capillary number is $10^{-4}$; the discrepancy at larger $\mathrm{Ca}$ is mostly because of the asymptotic nature of the model that becomes less accurate for increasing $\mathrm{Ca}$. At larger capillary numbers, we compare the uniform film thickness with the FEM-based numerical results of Ref. [19] for a bubble, showing perfect agreement in Fig. 5, agreements for the front and rear plane curvatures are also observed and are not reported here.

For viscosity ratios $\lambda>0$, we have validated our setup against the results from an axisymmetric boundary integral method (BIM) solver [36] for a droplet with $C a_{\infty}=0.05$ of viscosity ratios $\lambda=0.1$ and 10 , again exhibiting perfect agreement as displayed in Fig. 6

Based on the carefully performed validations against the theory, numerical results from FEM and BIM solvers, we are confident that the developed COMSOL implementation can be used to carry out high-fidelity two-phase simulations efficiently, at least for the 2D and 3D-axisymmetric configurations.

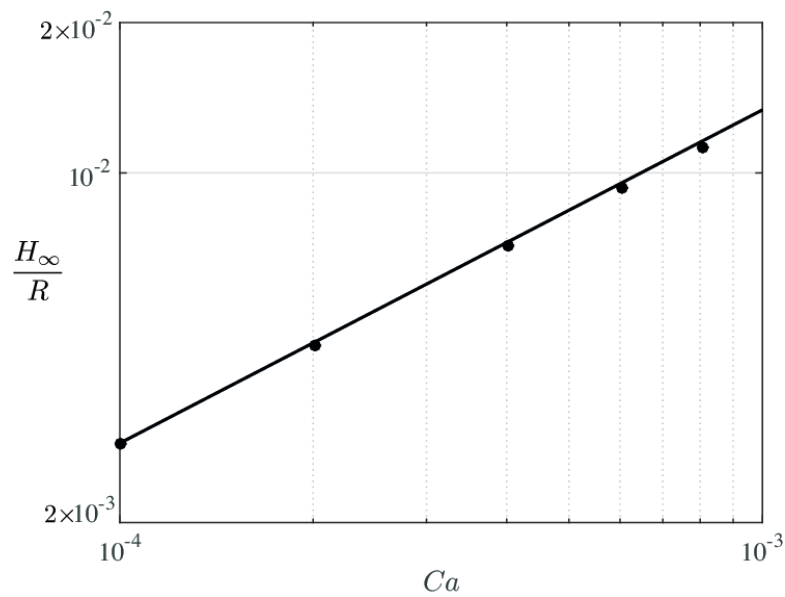

Fig. 4 Comparison between the uniform film thickness between the wall and a bubble obtained by the FEM-ALE simulations (symbols) and that predicted by Bretherton [9] (solid line) for the planar channel.

\section{Flow field}

3.1 Velocity profiles in the thin-film region

For a sufficiently long droplet/bubble, a certain portion of the lubrication film is of uniform thickness $H_{\infty}$ [9] (see Fig. 2 and Fig. 8). Within this portion, the velocity field both inside and outside the droplet is invariant in the streamwise

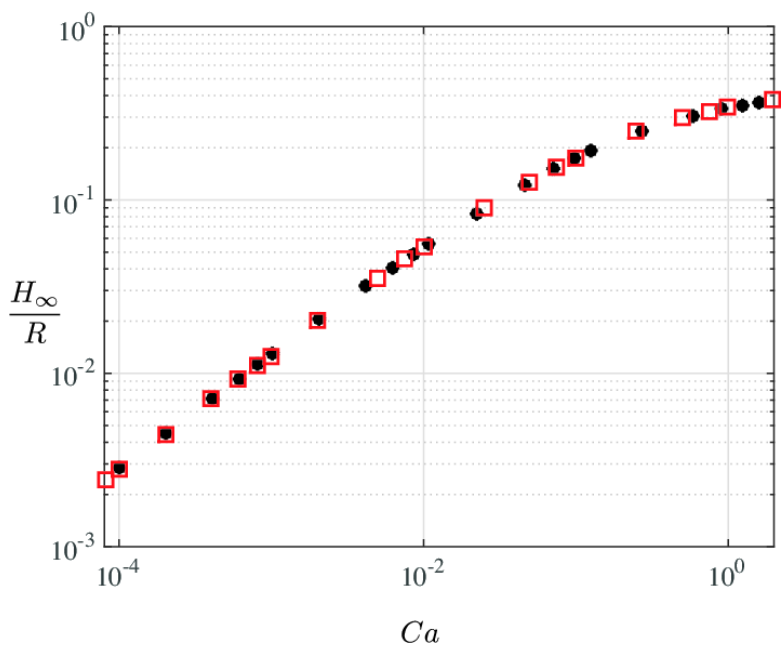

Fig. 5 Comparison between the uniform film thickness between the wall and a bubble obtained by the FEM-ALE simulations (full black circles) and that of Ref. [19] (empty red squares) for the planar channel.

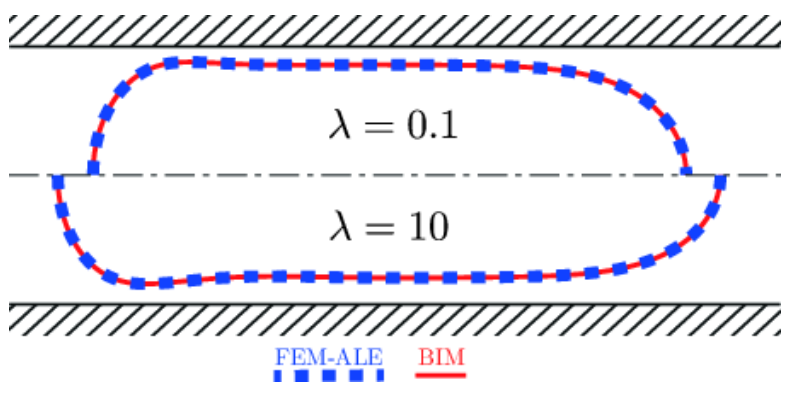

Fig. 6 Comparison between the droplet profiles obtained by the FEMALE computations (blue dashed) and the BIM (red solid) computations of Ref. [36] for an axisymmetric droplet in a tube with $C a_{\infty}=0.05$ of viscosity ratios $\lambda=0.1$ (upper half domain) and $\lambda=10$ (lower half domain).

direction and resembles the well known bi-Poiseuille profile that typically arises in several interfacial flows, for example a coaxial jet [27] (see Fig. 7). For $\lambda \ll 1$, the velocity profile in the film is almost linear, whereas for $\lambda \gg 1$, the velocity inside of the droplet is almost constant (pluglike profile). Nevertheless, the parabolic component of these profiles is crucial for the accurate prediction of the droplet velocity (see Sec. 5).

Assuming the bi-Poiseuille velocity profile, we describe the streamwise velocity $u_{i}(r)$ inside and $u_{o}(r)$ outside the droplet as a function of the off-centerline distance $r$ as:

$u_{i}(r)=\frac{1}{4 \mu_{i}} \frac{d p_{i}}{d z} r^{2}+A_{i} \ln r+B_{i}$,

$u_{o}(r)=\frac{1}{4 \mu_{o}} \frac{d p_{o}}{d z} r^{2}+A_{o} \ln r+B_{o}$,

where $p_{i}$ and $p_{o}$ are the inner, respectively outer, pressures, and $A_{i}, B_{i}, A_{o}$ and $B_{o}$ are undetermined constants. Given the finiteness of $u_{i}(r)$ at $r=0$, we have $A_{i}=0$. By satisfying the no-slip boundary condition on the channel walls $u_{o}(R)=$ 


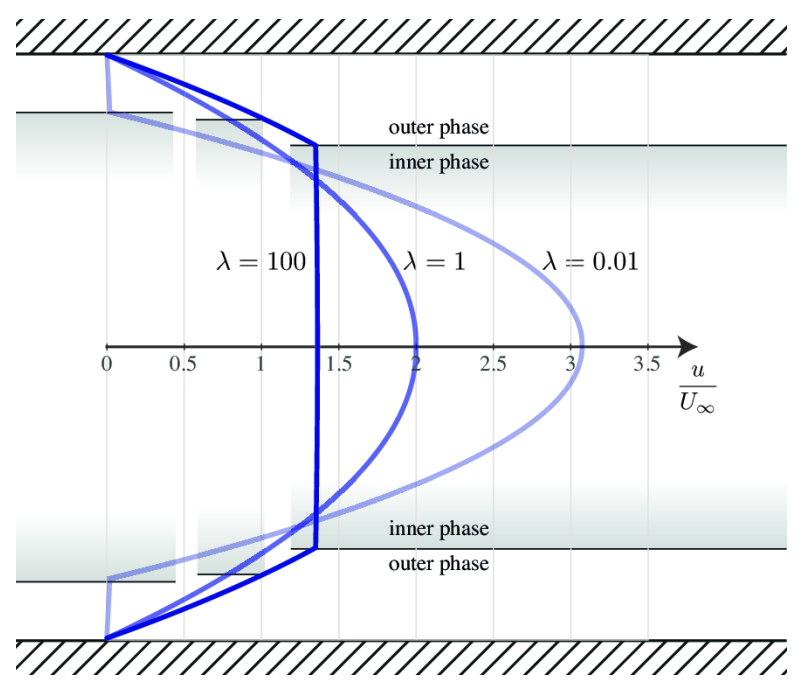

Fig. 7 Inner and outer phase velocity profiles in the uniform film region of an axisymmetric droplet with $C a_{\infty}=0.1$ and viscosity ratios $\lambda=0.01,1$ and 100 , represented in the laboratory frame.

$-U_{d}$, the continuity of velocities and tangential stresses on the interface $r=\tilde{r}=R-H$, namely, $u_{i}(\tilde{r})=u_{o}(\tilde{r})$ and

$\left.\mu_{i} \frac{d u_{i}}{d z}\right|_{r=\tilde{r}}=\left.\mu_{o} \frac{d u_{o}}{d z}\right|_{r=\tilde{r}}$,

we obtain the remaining constants

$$
\begin{aligned}
A_{o}= & \frac{1}{2 \mu_{o}}\left(\frac{d p_{i}}{d z}-\frac{d p_{o}}{d z}\right) \tilde{r}^{2}, \\
B_{i}= & -\frac{1}{4 \mu_{i} \mu_{o}}\left[\frac{d p_{o}}{d z}\left(R^{2}-\tilde{r}^{2}\right) \mu_{i}+\frac{d p_{i}}{d z} \tilde{r}^{2} \mu_{o}\right. \\
& \left.+2\left(\frac{d p_{i}}{d z}-\frac{d p_{o}}{d z}\right) \tilde{r}^{2} \mu_{i} \ln \left(\frac{R}{\tilde{r}}\right)\right]-U_{d} \\
B_{o}= & -\frac{1}{4 \mu_{o}}\left[\frac{d p_{o}}{d z} R^{2}+2\left(\frac{d p_{i}}{d z}-\frac{d p_{o}}{d z}\right) \tilde{r}^{2} \ln R\right]-U_{d} .
\end{aligned}
$$

Under the assumption of a slowly evolving film thickness, this velocity profile also holds in the nearby regions, where the thickness is $H$ rather than $H_{\infty}$. The derivation for the planar geometry is given in Appendix A.

\subsection{Recirculating flow patterns}

The axisymmetric velocity profile in the channel away from the droplet, in its moving reference frame, is given by

$u_{\infty}(r)=2 U_{\infty}\left[1-\left(\frac{r}{R}\right)^{2}\right]-U_{d}$.

As will become clear in Sec. 5 the droplet velocity can be obtained by imposing mass conservation. For the particular case of a bubble with $\lambda=0$, mass conservation reads $\left(U_{\infty}-\right.$ $\left.U_{d}\right) \pi R^{2}=-\pi\left[R^{2}-\left(R-H_{\infty}\right)^{2}\right] U_{d}$ [54], yielding $U_{\infty} / U_{d}=$
$\left(1-H_{\infty} / R\right)^{2}$. The velocity profile (11) can therefore be expressed as a function of $H_{\infty} / R$. As pointed out by Giavedoni \& Saita [19], when $\lambda=0$, the velocity at the centerline $u_{\infty}(r=0)$ in the bubble frame changes sign when $H_{\infty}=$ $H_{\infty}^{\star}=(1-1 / \sqrt{2}) R$. As a consequence, when the uniform film thicknesses is below $H_{\infty}^{\star}, u_{\infty}(0)>0$, an external recirculating flow pattern forms ahead of and behind the bubble. For the planar configuration, the critical film thickness for the appearance of the flow recirculation is $H_{\infty}^{\star}=R / 3$.

Based on the flow profiles derived in Sec. 3 and mass conservation (see Sec.5), we can generalize the critical thickness $H_{\infty}^{\star}$ to non-vanishing viscosity ratios $(\lambda>0)$ as (see Appendix $\mathrm{D}$ for the derivations):

$\frac{H_{\infty}^{\star}}{R}=1-\sqrt{\frac{(\lambda-1)(2 \lambda-1)}{2}} \frac{1}{\lambda-1}$

for the axisymmetric case and

$\frac{H_{\infty}^{\star}}{R}=\frac{1}{3} \frac{1}{1-\lambda}$

for the planar case. $H_{\infty}^{\star} / R$ reaches the value of 1 when $\lambda=$ $1 / 2$ or $\lambda=2 / 3$, for the axisymmetric and planar configuration, respectively. Nevertheless, the uniform film thickness is always much smaller than the channel half-width, $H_{\infty} \ll R$. For a fixed volume of fluid, large film thicknesses would correspond to long droplets, which might be unstable to the Rayleigh-Plateau instability as discussed in Sec. 2.1 .

For viscosity ratios $\lambda \geq 1 / 2(\lambda \geq 2 / 3)$ for the axisymmetric (planar) configuration, there is no critical film thickness above which the recirculation zones disappear, meaning that a recirculation region always exists for any capillary number.

At low capillary numbers, when the film thickness is below $H_{\infty}^{\star}$, the external recirculating flows are strong enough to induce the recirculation inside the droplet as well. Consequently, besides the two droplet vertices as permanent stagnation points (blue circles in Fig. 8), two stagnation rings emerge on the front and rear part of the axisymmetric interface (red stars in Fig. 8); likewise, four stagnation points arise in the planar case. The stagnation rings/points on the dynamic meniscus (red stars in Fig. 8) move outwards to the droplet vertices when $\mathrm{Ca}$ increases. When $H_{\infty}>H_{\infty}^{\star}$, these stagnation rings/points disappear, taking away with the recirculation regions accordingly (see Fig. 8,b)). Only the stagnation points at the droplet vertices remain.

However, since the stagnation rings/points at the droplet interface move outwards to the front and rear extremities when the film thickness increases with the capillary number, the recirculation regions might eventually detach from the interface before the critical film thickness $H_{\infty}^{\star}$ is reached. Nevertheless, since recirculation regions exist far away from the droplet as long as $H_{\infty}<H_{\infty}^{\star}$, another flow pattern must exist close to the droplet interface. The detached stagnation 


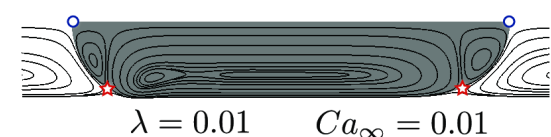

(a)

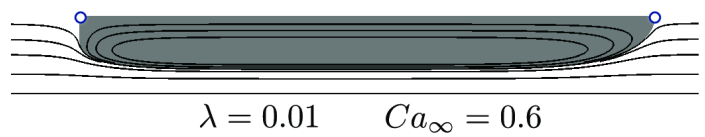

(b)

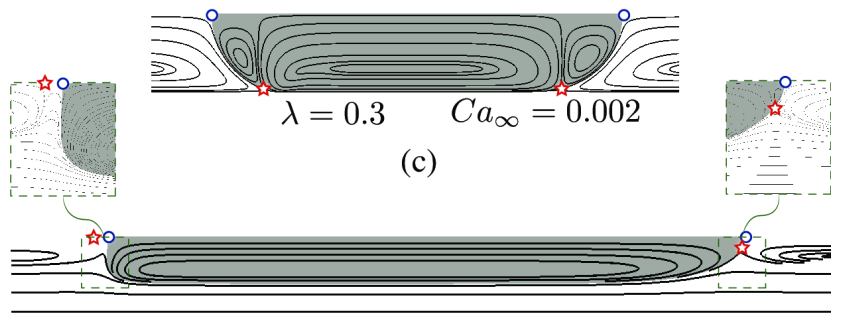

$\lambda=0.3 \quad C a_{\infty}=0.6$

(d)

Fig. 8 Streamlines and recirculation patterns for an axisymmetric droplet with different capillary numbers $C a_{\infty}$ and viscosity ratios $\lambda$ in a frame of reference moving with the droplet. The results are obtained from FEM-ALE numerical simulations. The permanent stagnation points at the droplet vertices are highlighted by blue circles, whereas the $(\mathrm{Ca}, \lambda)$-dependent stagnation rings/points are highlighted by red stars. Insets: detailed flow pattern at the droplet front and rear for $\lambda=0.3$ and $C a_{\infty}=0.6$.

points are located on the centerline outside the droplet, and no recirculating flow is present in the region between the detached stagnation point and the one at the droplet vertex (see rear of droplet in Fig. 8,d)). Contrasting with the case of Fig. 8.c), where the stagnation points on the dynamic meniscus induce recirculating flow inside the droplet at both front and rear parts, the inner rear recirculation zone disappears when the capillary number increases, as shown in Fig. 8(d). This indicates that the detachment of the recirculation region is not fore-aft symmetric. There is a large range of parameters for which a rear stagnation point is not at the droplet interface anymore and thus there is no recirculation inside the rear part of the droplet. We have found that the critical film thickness for which the stagnation ring/point at the rear detaches from the droplet interface corresponds to when the rear plane curvature of the droplet changes the sign (see also Sec. 7.1). For both the flow patterns as well as for the plane curvatures, the fore-aft asymmetry increases with the capillary number.

The phase diagram with the main different types of flow patterns as a function of the viscosity ratio $\lambda$ and film thickness $H_{\infty} / R$ is shown in Fig. 9 . Note that for viscosity ratios $\lambda \geq 1 / 2(\lambda \geq 2 / 3)$, the recirculation regions will be attached or detached from or to the droplet interface depending on the the uniform film thickness. Other very peculiar flow fields,

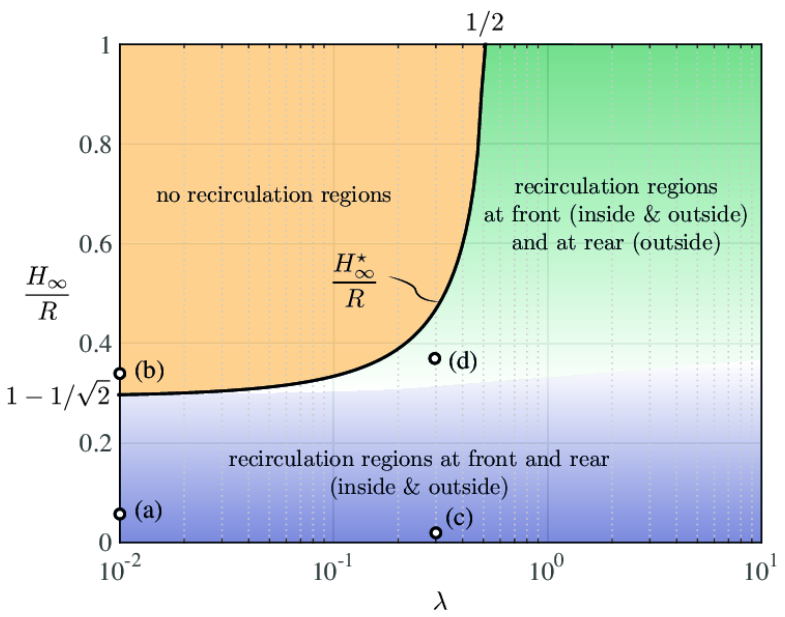

Fig. 9 Diagram of the main possible flow patterns for the axisymmetric configuration. The streamlines corresponding to the points (a)-(d) are shown in Fig. 8

as a detached finite recirculation region at the rear or a detached recirculation region at the front as observed by Giavedoni \& Saita [19,20], can be obtained for some parameter combinations. However, since the flow field structures are not the main aim of this work, an extended parametric study to detect all possible patterns has not been performed. Nevertheless, our current results do not validate all the flow patterns predicted in Ref. [28] based on asymptotic arguments, which indeed have not been verified neither experimentally nor numerically.

The flow fields for the planar configuration are not presented here as they are similar to those for the axisymmetric configuration.

\section{Film thickness}

\subsection{Asymptotic result in the low-Ca limit}

By following the work of Schwartz et al. [52], we derive an implicit expression predicting the thickness $H_{\infty}$ of the uniformly-thick lubrication film in the low- $\mathrm{Ca}$ limit when $H / R \ll 1$ satisfies. The derivation of the axisymmetric case is presented below, see Appendix B for the planar case. 
The flow rates at any axial location where the external film thickness is $H$ are:

$$
\begin{aligned}
q_{i} & =2 \pi \int_{0}^{R-H} u_{i}(r) r d r \\
& =-\pi(R-H)^{2}\left\{U_{d}+\frac{1}{8 \mu_{i} \mu_{o}}\left[2 \frac{d p_{o}}{d z} H(2 R-H) \mu_{i}\right.\right. \\
& +\frac{d p_{i}}{d z}(R-H)^{2} \mu_{o} \\
& \left.\left.+4\left(\frac{d p_{i}}{d z}-\frac{d p_{o}}{d z}\right)(R-H)^{2} \mu_{i} \ln \left(\frac{R}{R-H}\right)\right]\right\} \\
q_{o} & =2 \pi \int_{R-H}^{R} u_{o}(r) r d r \\
& =-\frac{\pi}{8 \mu_{o}}\left\{H ( 2 R - H ) \left[H^{2}\left(2 \frac{d p_{i}}{d z}-3 \frac{d p_{o}}{d z}\right)\right.\right. \\
& \left.+2\left(\frac{d p_{i}}{d z}-\frac{d p_{o}}{d z}\right) R^{2}-H\left(4 \frac{d p_{i}}{d z}-6 \frac{d p_{o}}{d z}\right) R\right] \\
& \left.+4\left(\frac{d p_{i}}{d z}-\frac{d p_{o}}{d z}\right)(R-H)^{4} \ln \left(\frac{R}{R-H}\right)\right\} \\
& -\pi H(2 R-H) U_{d} .
\end{aligned}
$$

Assuming that $H / R \ll 1$, the volumetric fluxes up to the second order are

$$
q_{i} \approx-\pi R^{2}\left(U_{d}+\frac{1}{8 \mu_{i}} \frac{d p_{i}}{d z} R^{2}+\frac{1}{2 \mu_{o}} \frac{d p_{i}}{d z} R H+\frac{1}{2 \mu_{o}} \frac{d p_{o}}{d z} H^{2}\right)
$$$$
q_{o} \approx-2 \pi R H\left(U_{d}+\frac{1}{4 \mu_{o}} \frac{d p_{i}}{d z} H R+\frac{1}{3 \mu_{o}} \frac{d p_{o}}{d z} H^{2}\right) .
$$

In the droplet frame, the inner flow rate is $q_{i}=0$. Furthermore, in the region with a uniformly-thick film, $H=H_{\infty}$; the inner and outer pressure gradient balances, $\frac{d p_{i}}{d z}=\frac{d p_{o}}{d z}$. Using these two conditions one can obtain the pressure gradient in the uniform film region

$\left.\frac{d p}{d z}\right|_{r=R-H_{\infty}} \approx-\frac{8 \mu_{i} U_{d}}{R^{2}+4 \lambda H_{\infty} R+4 \lambda H_{\infty}^{2}}$

and the outer flow rate in the $H_{\infty} / R \ll 1$ limit is

$$
\begin{aligned}
q_{o} & \approx-2 \pi R H_{\infty}\left[\frac{3 R^{2}+6 \lambda H_{\infty} R+4 \lambda H_{\infty}^{2}}{3\left(R^{2}+4 \lambda H_{\infty} R+4 \lambda H_{\infty}^{2}\right)}\right] U_{d} \\
& \approx-2 \pi R H_{\infty}\left(\frac{R+2 \lambda H_{\infty}}{R+4 \lambda H_{\infty}}\right) U_{d} .
\end{aligned}
$$

In the dynamic meniscus regions, the inner and outer pressure gradients are not equal and their difference is proportional to the mean curvature of the interface at $r=R-H$, through Laplace's law. By assuming a quasi-parallel flow whose radial velocity is weaker than the axial velocity by one order of magnitude and a small capillary number, the normal stress condition at the interface is not affected by viscous stresses. Hence, the axial gradient of the normal stress condition reads

$\frac{d p_{i}}{d z}-\frac{d p_{o}}{d z}=\gamma \frac{d^{3} H}{d z^{3}}$,

where the axial gradient of the curvature in the azimuthal direction is neglected as it is an order smaller. The pressure gradients $d p_{i} / d z$ and $d p_{o} / d z$ as functions of $H$ can be obtained from Eqs. (16), 17) imposing mass conservation, i.e. imposing $q_{i}=0$ and $q_{o}$ given by Eq. (19):

$$
\begin{aligned}
\frac{d p_{i}}{d z} & \approx \frac{4 \lambda\left(-6 H_{\infty}^{2} \lambda+4 H_{\infty} H \lambda-3 H_{\infty} R+H R\right) \mu_{o} U_{d}}{H R\left(4 H_{\infty} \lambda+R\right)(H \lambda+R)}, \\
\frac{d p_{o}}{d z} & \approx \frac{3\left(H_{\infty}-H\right)\left[8 H_{\infty} H \lambda^{2}+2\left(H_{\infty}+H\right) \lambda R+R^{2}\right] \mu_{o} U_{d}}{H^{3}\left(4 H_{\infty} \lambda+R\right)(H \lambda+R)} .
\end{aligned}
$$

By plugging Eqs. 21), 22) into Eq. 20, and adopting the change of variables $H=H_{\infty} \eta$ and $z=H_{\infty}(3 C a)^{-1 / 3} \xi$ in the spirit of Bretherton [9], we obtain an universal governing equation for the scaled film thickness $\eta$ when taking the limit $H_{\infty} / R \rightarrow 0$ :

$\frac{d^{3} \eta}{d \xi^{3}}=\frac{\eta-1}{\eta^{3}}\left[\frac{1+2 m(1+\eta+4 m \eta)}{(1+4 m)(1+m \eta)}\right]$

where

$m=\lambda \frac{H_{\infty}}{R}$

denotes the rescaled viscosity ratio. The corresponding planar counterpart reads (see derivation in Appendix B)

$\frac{d^{3} \eta}{d \xi^{3}}=2 \frac{\eta-1}{\eta^{3}}\left[\frac{2+3 m(1+\eta+3 m \eta)}{(1+3 m)(4+3 m \eta)}\right]$.

If the limit of vanishing uniform film thickness is not considered, the resulting equations for $\eta$ would depend on $H_{\infty} / R$ [50]. In the limit of $m \rightarrow 0$, the classical Landau-LevichDerjaguin equation [13,38] is retrieved for both configurations. Following Bretherton [9], Eqs. [23) and (24) can be integrated to find the uniform film thickness $H_{\infty} / R$ (see also Cantat [10] for more details).

First, the equations can be linearized in the uniform film region around $\eta \approx 1$, giving

$\frac{d^{3} \eta}{d \xi^{3}}=K(\eta-1)$

where $K$ is a constant depending on the geometrical configurations and the viscosity ratio $m$. Equation 25 has a monotonically increasing solution with respect to $\xi$ for the front dynamic meniscus, $\xi \rightarrow \infty$, and an oscillatorily increasing solution for the rear, $\xi \rightarrow-\infty$, as derived in Sec. 6. The solution of the front meniscus is $\eta(\xi)=1+\alpha \exp \left(K^{1 / 3} \xi\right)$, where $\alpha$ is a small parameter, typically $10^{-6}$. Second, the 
nonlinear equations (23) and 24) can be integrated numerically as an initial value problem with a fourth-order RungeKutta scheme, starting from the linear solution until the plane curvature of the interface profile becomes constant. A region of constant plane curvature, called static meniscus region (see Fig. 1) exists as $d^{3} \eta / d \xi^{3} \approx 0$ for $\eta \gg 1$ (see red line on Fig. 11). In the static meniscus region, the interface profile is a parabola: $\eta=P \xi^{2} / 2+C \xi+D$, or, in terms of film thickness, $H=P(3 C a)^{2 / 3} z^{2} /\left(2 H_{\infty}\right)+C(3 C a)^{1 / 3} z+D H_{\infty}$, where $P, C$ and $D$ are real-valued constants. Thus, $P$ is set by the constant plane curvature obtained by the integration of the nonlinear equation.

The procedure can be repeated for any rescaled viscosity ratio $m$ and the obtained results for the coefficient $P$ can well described by the fitting law [52]:

$P(m)=\frac{0.643}{2}\left\{1+2^{2 / 3}+\left(2^{2 / 3}-1\right) \tanh \left[1.2 \log _{10} m+c_{1}\right]\right\}$

where the constant $c_{1}=0.1657$ for the axisymmetric configuration and $c_{1}=0.0159$ for the planar configuration (see Fig. 10. The well known limits for a bubble $P(0)=0.643$ [9] and a very viscous droplet $P(m \rightarrow \infty)=2^{2 / 3} P(0)$ [10] are recovered.

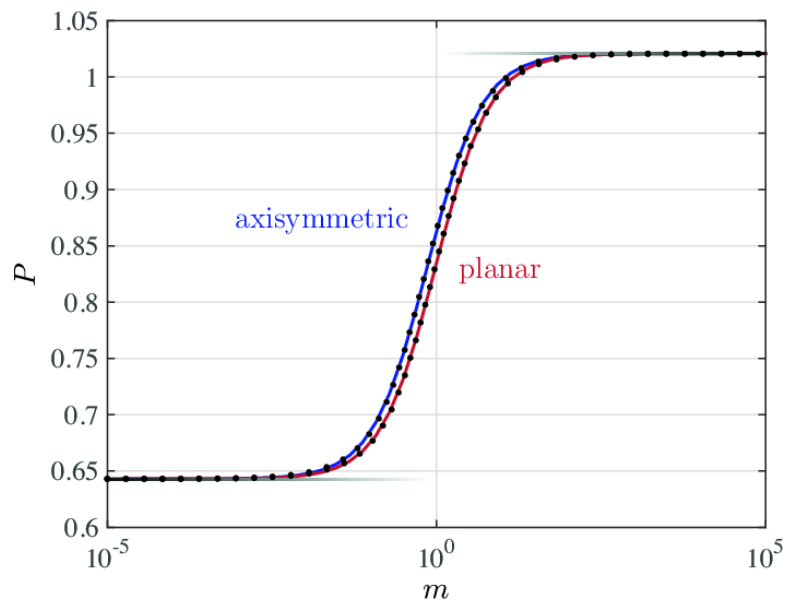

Fig. 10 Film-thickness coefficient $P$ obtained for discrete $m$ values (symbols) and fitting law (26) (solid lines) as a function of the rescaled viscosity ratio $m$.

To obtain the uniform film thickness, the matching principle proposed by Bretherton [9] is employed. The plane curvature $\kappa=d^{2} H / d z^{2}=P(3 C a)^{2 / 3} / H_{\infty}$ in the static region has to match that of the front hemispherical cap of radius $R$, which exists for small capillary numbers (see red dashed line in Fig. 11). A rigorous asymptotic matching can be found in Park \& Homsy [46] for a bubble with $m=0$. When $m \neq 0$, the coefficient $P(m)$ depends implicitly on $H_{\infty}$, and thus on $\mathrm{Ca}$, through $\mathrm{m}$, leading to an implicit asymptotic relation for

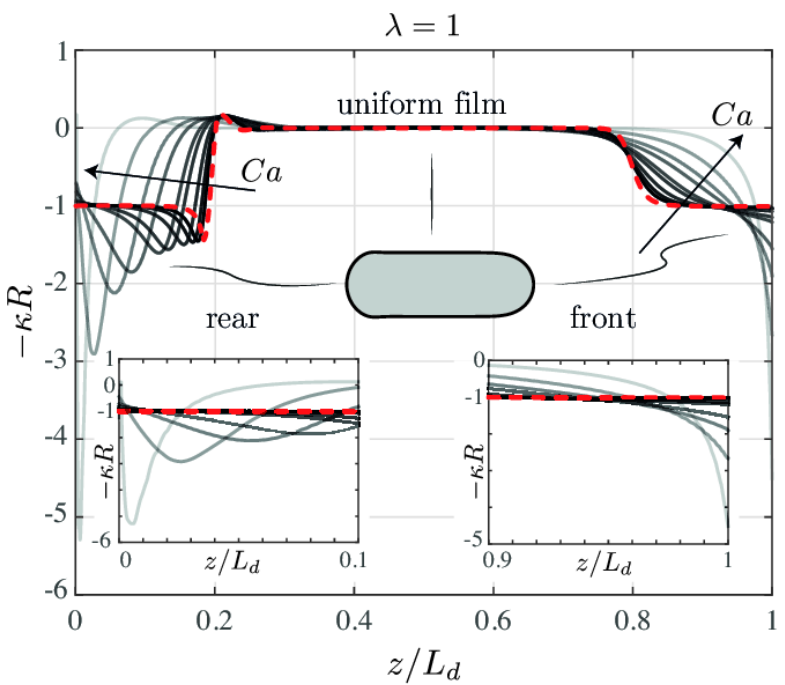

Fig. 11 Plane curvature of the droplet interface for exponentially increasing capillary numbers in the range $10^{-4}<C a<1$, obtained from FEM-ALE numerical simulations where $\lambda=1$. The dashed red line is for the smallest $\mathrm{Ca}$. The $z$ axis is rescaled by the droplet length to facilitate the comparison. Insets: zoom-in on the front and rear menisci. Similar profiles are obtained for other viscosity ratios.

$H_{\infty} / R$ as:

$\frac{H_{\infty}}{R}=P(m)(3 C a)^{2 / 3}$.

Strictly speaking, the uniform film thickness of viscous droplets $(\lambda \neq 0)$ in the low $C a$ limit does not scale with $C a^{2 / 3}$ as for a bubble $(\lambda=0)$.

\subsection{Empirical model in the low-Ca limit}

Equation 27 holds for capillary numbers as low as below $10^{-3}$ [9]. We solve Eq. (27) numerically and present the coefficient $P$ and the uniform film thickness $H_{\infty} / R$ versus $C a$ in Fig. 12 for the axisymmetric case. In order to derive an explicit formulation to predict the film thickness in this $\mathrm{Ca}$ regime, we define $\bar{P}$ as a $C a$-averaged value of $P$ and define the empirical model

$\frac{H_{\infty}}{R}=\bar{P}(\lambda)(3 C a)^{2 / 3}$

where $\bar{P}(\lambda)$ is independent of $C a$ (see dashed lines in Fig. 12(a) and can be approximated by the fitting law (see Fig. 13):

$$
\begin{array}{r}
\bar{P}(\lambda)=\frac{0.643}{2}\left\{1+2^{2 / 3}\right. \\
\left.+\left(2^{2 / 3}-1\right) \tanh \left[1.28 \log _{10} \lambda+c_{2}\right]\right\} .
\end{array}
$$

where the constant $c_{2}=-2.36$ for the axisymmetric case and $c_{2}=-2.52$ for the planar case are obtained by fitting. For $\lambda=0, \bar{P}=0.643$ is recovered and $H_{\infty} / R$ indeed scales with $C a^{2 / 3}$, at least when $C a<10^{-3}$. Figure 12(b) also shows 
that the empirically obtained film thickness (dashed lines) Eq. (28) agrees reasonably well with the FEM-ALE simulation results (symbols), whereas the implicit law (solid lines) Eq. (27) slightly underestimates them at very low $\mathrm{Ca}$. To cure this mismatch, Hodges et al. [28] proposed a modified interface condition, which however is found to overestimate the thickness more than that underestimated by the original implicit law.

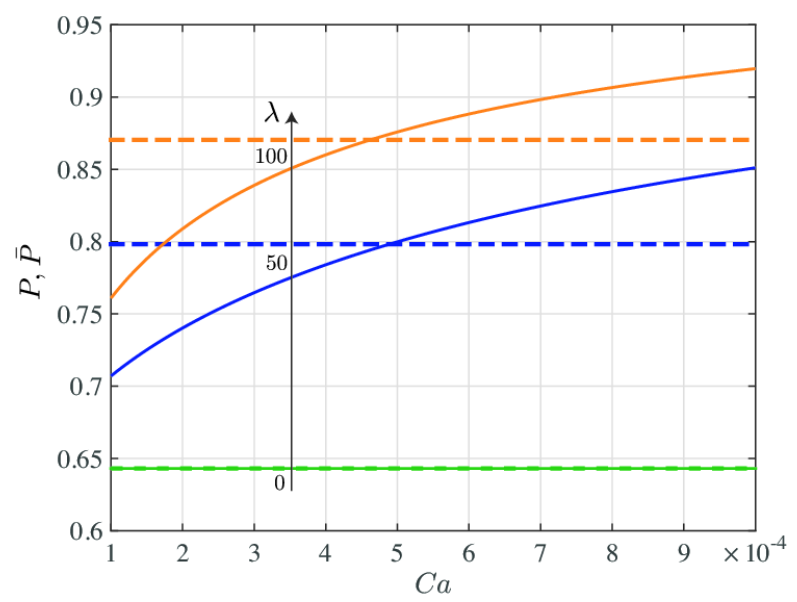

(a)

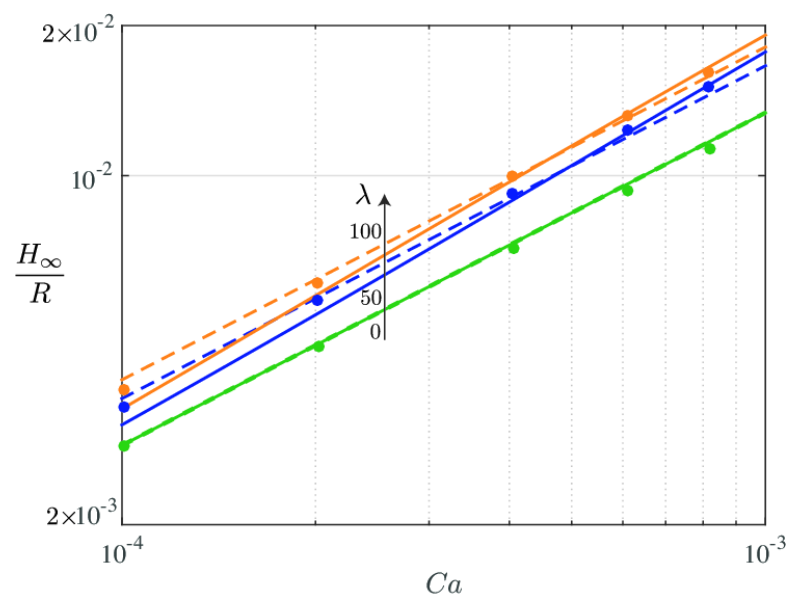

(b)

Fig. 12 (a) Coefficient $P$ (solid lines) obtained by solving Eq. 27] and the mean coefficient $\bar{P}$ (dashed lines) for the axisymmetric configuration. The viscosity ratios are $\lambda=0,50$ and 100. (b) The uniform film thickness $H_{\infty} / R$ from Eq. 27] (solid lines) and Eq. 28) (dashed lines), compared to the FEM-ALE simulation results (symbols).

\subsection{Model for $10^{-3} \lesssim C a \lesssim 1$}

Despite the explicit law for the uniform-film thickness prediction with $\bar{P}$ proved to be satisfactory, its validity range is restricted to low capillary numbers. As known since the experiments of Taylor [56], the film thickness of a bubble sat-

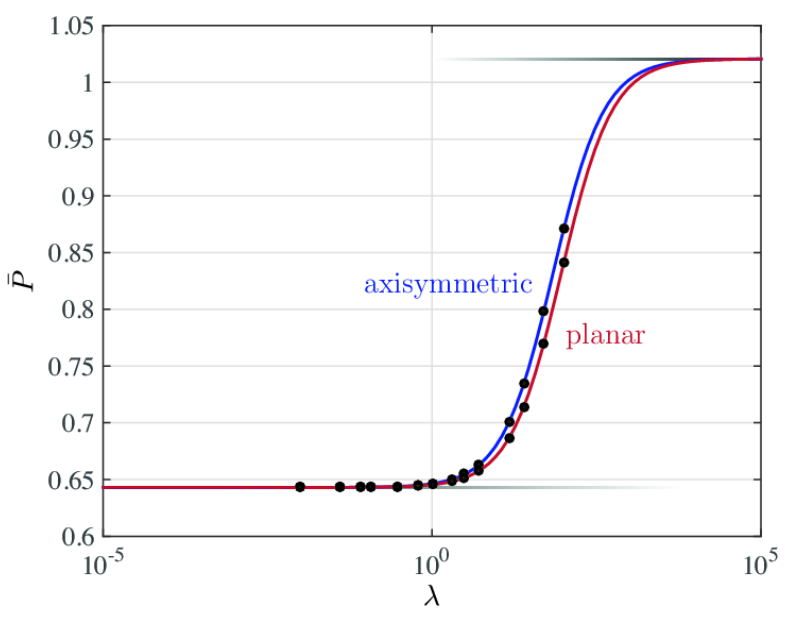

Fig. 13 Mean coefficient $\bar{P}$ (symbols) obtained by $\mathrm{Ca}$-averaging the results of the implicit relation Eq. 27) and fitting law (solid lines) Eq. (29) versus the viscosity ratio $\lambda$.

urates for increasing $\mathrm{Ca}$. Aussillous and Quéré [5] proposed a model for $\lambda=0$, which agrees well with the experimental data of [56], further inspiring the two very recent works of Refs. [33, 12]. In the same vain, we propose an empirical model for the film thickness $H_{\infty}$ as a function of both $\mathrm{Ca}$ and $\lambda$

$\frac{H_{\infty}}{R}=\frac{\bar{P}(\lambda)(3 C a)^{2 / 3}}{1+\bar{P}(\lambda) Q(\lambda)(3 C a)^{2 / 3}}$,

where the coefficient $Q$ is obtained by fitting Eq. (30) to the database constructed from our extensive FEM-ALE simulations over a broad range of $\mathrm{Ca}$ for different $\lambda$. The proposed function of $Q(\lambda)$ is given in Appendix $\mathrm{E}$ and plotted in Fig. 14. For an axisymmetric bubble, we find $Q=2.48$, in accordance with the estimation $Q=2.5$ of Ref. [5]. We now present in Fig. 15 the numerical film thickness (symbols) and the empirical model (lines) for $\lambda=1$. For the sake of clarity, the results for $\lambda=0$ and 100 are shown in the appendix Fon Fig. 25. For $\lambda=1$, the thickness of the two configurations coincide. However, when $C a \sim O(1)$, the film is thicker in the planar configuration than in the axisymmetric one for a bubble $(\lambda=0)$; the trend reverses for a highlyviscous droplet $(\lambda=100)$. This $\lambda$-dependence of the film thickness is indeed implied by the crossover of the two fitting functions $Q(\lambda)$ at $\lambda=1$ shown in Fig. 14 .

It has to be noted that when the capillary number is increased, the regions of constant plane curvature in the static front and rear caps reduce in size and eventually disappear (see Fig. 11), and this for all viscosity ratios. The matching to a region of constant plane curvature for large capillary numbers as proposed by Refs. [33, 12] might be questionable for this $\mathrm{Ca}$-range.

The uniform film thickness of droplets with $37 \%$ and $82 \%$ larger volume, resulting in longer droplets, are com- 


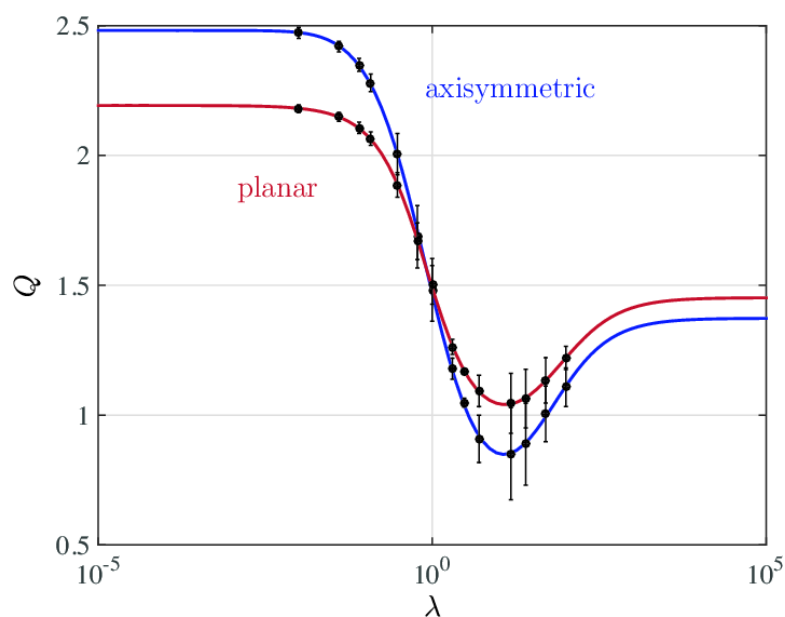

Fig. 14 Coefficient $Q$ obtained for the simulated viscosity ratios (symbols) and proposed fitting law (see Appendix E) as a function of the viscosity ratio $\lambda$.

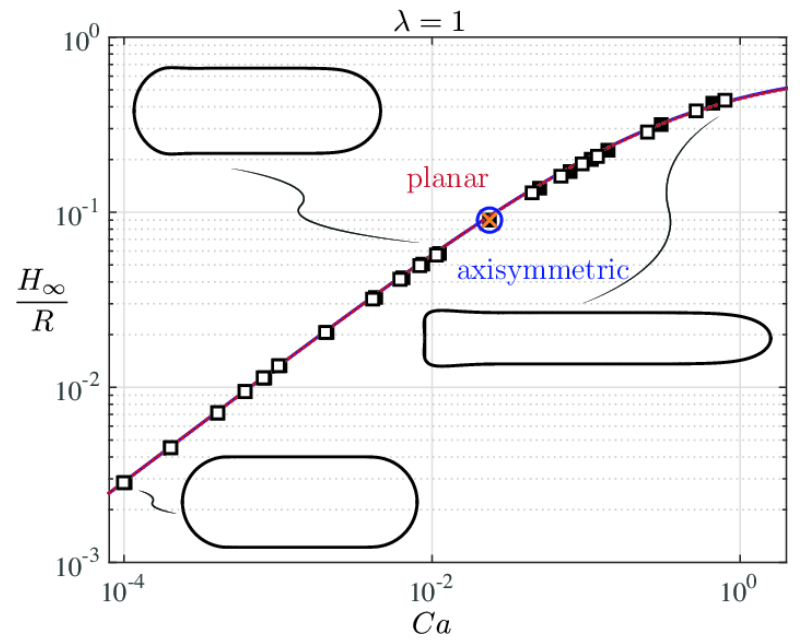

(a)

Fig. 15 Uniform film thickness given by Eq. 30] (lines) and FEMALE numerical results (symbols) as a function of the droplet capillary number for $\lambda=1$ and both axisymmetric (blue solid line, full symbols) and planar (dashed red line, empty symbols) geometries. Cross and circle correspond to a droplet with $37 \%$ and $82 \%$, respectively, larger volume than the standard one used for the axisymmetric geometry.

pared on Fig. 15, showing that as long as such a uniform region exists, the results are independent of the droplet length.

\section{Droplet velocity}

Equipped with the model of the uniform-film thickness $H_{\infty}$, we derive the droplet velocity based on the velocity profiles in the uniform-film region given in Sec. 3.1. At the location $H=H_{\infty}$ where the interface is flat, the pressure gradients are equal, $d p_{i} / d z=d p_{o} / d z=d p / d z$. We further use $q_{o}=$ $\pi R^{2}\left(U_{\infty}-U_{d}\right)$ imposed by mass conservation and $q_{i}=0$ (in the moving frame of the droplet) to obtain the analytical expressions for the pressure gradient

$$
\left.\frac{d p}{d z}\right|_{r=R-H_{\infty}}=\frac{-8 R^{2} U_{\infty} \mu_{i}}{\left(R-H_{\infty}\right)^{4}+H_{\infty}\left(2 R-H_{\infty}\right)\left(2 R^{2}-2 H_{\infty} R+H_{\infty}^{2}\right) \lambda},
$$

and the droplet velocity

$U_{d}=\frac{R^{2}\left[\left(R-H_{\infty}\right)^{2}+2 H_{\infty}\left(2 R-H_{\infty}\right) \lambda\right]}{\left(R-H_{\infty}\right)^{4}+H_{\infty}\left(2 R-H_{\infty}\right)\left(2 R^{2}-2 H_{\infty} R+H_{\infty}^{2}\right) \lambda} U_{\infty}$.

The relative velocity of the axisymmetric droplet with respect to the underlying velocity reads

$$
\frac{U_{d}-U_{\infty}}{U_{d}}=\frac{\left(2-\frac{H_{\infty}}{R}\right) \frac{H_{\infty}}{R}\left[1+\left(2-\frac{H_{\infty}}{R}\right) \frac{H_{\infty}}{R}(\lambda-1)\right]}{1+\left(2-\frac{H_{\infty}}{R}\right) \frac{H_{\infty}}{R}(2 \lambda-1)} .
$$

An analogous derivation for the planar configuration yields (see Appendix C):

$$
\frac{U_{d}-U_{\infty}}{U_{d}}=\frac{\frac{H_{\infty}}{R}\left\{2-\frac{H_{\infty}}{R}\left[4+2 \frac{H_{\infty}}{R}(\lambda-1)-3 \lambda\right]\right\}}{2+\left(2-\frac{H_{\infty}}{R}\right) \frac{H_{\infty}}{R}(3 \lambda-2)} .
$$

Eqs. (30) and (32) form a system of the two unknowns, namely the droplet capillary number $\mathrm{Ca}$ and the uniform film thickness $H_{\infty} / R$. It is important to remind that the former is related to the droplet velocity via $\mathrm{Ca}=\mathrm{Ca} a_{\infty} U_{d} / U_{\infty}$. For a given combination of inflow capillary number $C a_{\infty}$ and viscosity ratio $\lambda$ as the input, the system can be solved numerically (see Matlab file filmThicknessAndVelocity.m in the Supplementary Material) outputting $C a$ and $H_{\infty} / R$. The predicted relative velocity $\left(U_{d}-U_{\infty}\right) / U_{d}$ (lines) agrees well the FEM-ALE simulation results (symbols) as shown in Fig. 16

In the limit of $H_{\infty} / R \rightarrow 0$, the relative velocity can be approximated asymptotically as

$\frac{U_{d}-U_{\infty}}{U_{d}}=2\left(\frac{H_{\infty}}{R}\right)-(1+4 \lambda)\left(\frac{H_{\infty}}{R}\right)^{2}+O\left(\frac{H_{\infty}}{R}\right)^{3}$

for the axisymmetric case, and

$\frac{U_{d}-U_{\infty}}{U_{d}}=\left(\frac{H_{\infty}}{R}\right)-\frac{3 \lambda}{2}\left(\frac{H_{\infty}}{R}\right)^{2}+O\left(\frac{H_{\infty}}{R}\right)^{3}$

for the planar geometry. For very low capillary numbers, the asymptotic estimates predict that the relative droplet velocity scales with $H_{\infty} / R$, and hence with $C a^{2 / 3}$ [54]. The viscosity ratio $\lambda$ only enters at second order of $H_{\infty} / R$, which however influences the validity range of the asymptotic estimates 35 and 36 considerably. The asymptotic estimates are exact for $\lambda=0$. In this case, Eqs. 35 and 36 reduce to the well known predictions for bubbles $\left(2-H_{\infty} / R\right) H_{\infty} / R$ and $H_{\infty} / R[9,54,39$, respectively (see Fig. 16(a)]. For nonvanishing $\lambda$, the complete expressions 33 and 34 should be employed (see Fig. 16(b)). For example, the asymptotic estimate for $\lambda=100$ is only valid when $C a_{\infty}<10^{-4}$ (see Fig. 16(c)]. 


\section{Minimum film thickness}

At low capillary numbers $\mathrm{Ca}$, the droplet interface exhibits an oscillatory profile between the uniform thin film and the rear static cap (see Fig. 11). The minimum film thickness in the low $\mathrm{Ca}$ limit can be computed by integrating the lubrication equation (23) or (24) for $\xi=0$ to $\xi \rightarrow-\infty$. The initial condition for this initial value problem is given by the solution of the linear equation 25 for negative $\xi$ : $\eta=$ $1+\alpha \exp \left(-K^{1 / 3} \xi / 2\right) \cos \left(\sqrt{3} K^{1 / 3} \xi / 2+\phi\right)$, where $\alpha$ is a small parameter of order $10^{-6}$ and $\phi$ is a parameter taken such that the constant plane curvature of the nonlinear integrated solution at $\xi \rightarrow-\infty$ is equal to the one of the front static cap [9,10] as discussed in Sec. 4.1. Note that the linear solution for the rear dynamic meniscus presents oscillations. The minimum film thickness of the obtained profile is found to follow the empirical model [9]

$\frac{H_{\min }}{R}=F(m) P(m)(3 C a)^{2 / 3} \quad$ with $\quad m=\lambda \frac{H_{\infty}}{R}$,

where $F(m)$ is a coefficient obtained through fitting Eq. (37) to our numerical database (see Fig. 17(a) . Similar to the mean coefficient $\bar{P}$ adopted in Sec. 4, a $C a$-averaged $F(m)$ can be introduced as $\bar{F}$, that is further assumed as 0.716 in view of its very weak dependence on $\lambda$ shown in Fig. 17(b).

The minimum film thickness is bounded by the thickness of the uniform film and hence will saturate at large capillary numbers. Thus, for sufficiently large $C a$ values, the oscillations at the rear interface would disappear and $H_{\min }=H_{\infty}$. It is therefore natural to propose a rational function model of $H_{\min }$ for a broader $\mathrm{Ca}$-range as the one for $H_{\infty}$ :

$\frac{H_{\min }}{R}=\frac{\bar{P}(\lambda) \bar{F}(3 C a)^{2 / 3}}{1+\bar{P}(\lambda) \bar{F} G(\lambda)(3 C a)^{2 / 3}}$.

The above minimum film thickness model (38) together with the coefficient $G$ is in good agreement with the results of the numerical simulations (see Fig. 18 and Fig. 26). The proposed fitting of the coefficient $G$ as a function of the viscosity ratio (see Fig. 19) is given in Appendix E.

\section{Front and rear total stress jumps}

The dynamics of a translating bubble in a capillary tube has been characterized since the seminal work of Bretherton [9] not only by the mean and minimum film thickness, the relative velocity compared to the mean velocity, but also by the mean curvature of the front and rear static menisci. In fact, for $\mathrm{Ca} \rightarrow 0$, the pressure drop across the interface is directly related to the expression of its mean curvature via the Laplace law. Having generalized the film thickness and droplet velocity models for non-vanishing viscosity ratios, we are hereby focusing on the evolution of the plane curvature of the front and rear static caps versus the capillary number and viscosity ratio. The mean curvature at the droplet extremities is equivalent to the corresponding plane curvature for the planar configuration or to its double for the axisymmetric configuration: $\mathscr{C}_{f, r}=\chi \kappa_{f, r}$, with $\chi=2$ (resp. $\chi=1$ ) for the axisymmetric (resp. planar) configuration (see Sec. 2.2. As it will be shown, given the rather broad range of capillary numbers considered (approaching $O(1)$ ), it is insufficient to consider the interface mean curvature alone to provide an accurate prediction of the pressure drop, but the jump in the normal viscous stress has to be accounted for.

For the incompressible Newtonian fluids considered, the viscous stress tensor is $\tau=\mu\left[(\nabla \mathbf{u})+(\nabla \mathbf{u})^{T}\right]$, and hence the $z$-direction normal total stress $\sigma_{z z}$ is given by

$\sigma_{z z}=-p+\tau_{z z}=-p+2 \mu \frac{\partial u}{\partial z}$.

Applying the difference (between inner and outer phases) operator $\Delta$ to Eq. (39) and based on the dynamic boundary condition in the normal direction (4) at the droplet front and rear extremities, we get

$\Delta \sigma_{z z_{f, r}}=-\Delta p_{f, r}+\Delta \tau_{z z_{f, r}}=-\gamma \chi \kappa_{f, r}$

which indicates that the total stress jump at the front/rear extremities scales with the local interface mean curvature and is the sum of the pressure jump and the normal viscous stress jump. These quantities will be modeled separately in the following sections.

\subsection{Front and rear plane curvatures}

In the spirit of the empirical film thickness model, the plane curvature $\kappa_{f}$ of the front meniscus and that of the rear, $\kappa_{r}$, are approximated by the rational function model

$\kappa_{f, r} R=\frac{1+T_{f, r}(\lambda)(3 C a)^{2 / 3}}{1+Z_{f, r}(\lambda)(3 C a)^{2 / 3}}$,

where $T_{f, r}$ and $Z_{f, r}$ as $\lambda$-dependent constants are obtained by fitting Eq. 41] to the FEM-ALE data (see Appendix E). It is worth-noting that the asymptotic series of the proposed expression,

$\kappa_{f, r} R \sim 1+\left(T_{f, r}-Z_{f, r}\right)(3 C a)^{2 / 3}+O\left(C a^{4 / 3}\right)$,

is in line with the law proposed by Bretherton [9], namely $1+\beta_{f, r}(3 \mathrm{Ca})^{2 / 3}+O\left(\mathrm{Ca}^{4 / 3}\right)$. Thus, the empirical model (41), which is in excellent agreement with the numerical results (see Fig. 20 and 21 as well as Fig. 27 and 28), can be regarded as an empirical extension of Bretherton's law to a broader capillary numbers range up to 1 . The mean curvature at the droplet extremities is given by $\mathscr{C}_{f, r}=\chi \kappa_{f, r}$. 
7.2 Front and rear pressure jumps - classical model

Following the literature [9,12], the dimensionless pressure jump $\Delta p_{f, r} R / \gamma=\left(p_{f, r}^{i}-p_{f, r}^{o}\right) R / \gamma$ at the front and rear of the droplet is described by the empirical model

$$
\frac{\Delta p_{f, r} R}{\gamma}=\chi\left[1+S_{f, r}(\lambda)(3 C a)^{2 / 3}\right]
$$

where $\chi=2$ (resp. $\chi=1$ ) for the axisymmetric (resp. planar), and $S_{f, r}$ is a $\lambda$-dependent coefficient. Equation (43) is in fact inspired by the curvature model proposed by Bretherton [9] exploiting the Laplace law [12], reason why we call it classical model. The coefficient $S_{f, r}$ could be derived from the integration of the lubrication equation. (23) or 24, which is valid in the low- $\mathrm{Ca}$ limit when the viscous stresses and their jumps are negligible. To broaden the $C a$ range of the model, we obtain $S_{f, r}$ through fitting to the FEM-ALE data. Nevertheless, as visible in Fig. 22 and Fig. 23, the model fails to precisely describe the numerical data, particularly for the rear pressure jump at high $\mathrm{Ca}$ values (see Fig. 23.

After explaining our model for the normal viscous stress jump in Sec. 7.3. we will show in Sec. 7.4 that the pressure jump can be better approximated by summing up the two contributions from the interface mean curvature and the normal viscous stress jump, which are modeled separately. The importance of the normal viscous stress jump for the pressure jump is already noticeable when comparing the evolutions of the plane curvature $\kappa_{f, r}$ and the one of the pressure jump $\Delta p_{f, r} / \gamma$ in Figs. 20 and 22 or in Figs. 21 and 23

\subsection{Front and rear normal viscous stress jumps}

The dimensionless normal viscous stress jump $\Delta \tau_{z z} R / \gamma=$ $\left(\tau_{z z_{f, r}}^{i}-\tau_{z z_{f, r}}^{o}\right) R / \gamma$ at the front and rear of the droplet is approximated by the following model

$$
\frac{\Delta \tau_{z z_{f, r}} R}{\gamma}=\frac{M_{f, r}(\lambda)(3 C a)+N_{f, r}(\lambda)(3 C a)^{4 / 3}}{1+O_{f, r}(\lambda)(3 C a)},
$$

where $M_{f, r}, N_{f, r}$ and $O_{f, r}$ are viscosity ratio dependent coefficients found by fitting Eq. (44) to the FEM-ALE data. The normal viscous stress jumps indeed scale with $C a$ for small capillary numbers, as found by Bretherton [9]. The comparison between the model and the numerical results is shown in the insets of Figs. 22 and 23 , where the results for $\lambda=0$ are shown. The results for $\lambda=1$ and 100 can be found in Figs. 29 and 30 . The stress jump $\Delta \tau_{z z}$ is found to be small in the case of $\lambda=1$ and it varies with $C a$ non-monotonically for the other viscosities.

\subsection{Front and rear pressure jumps - improved model}

Using the dynamic boundary condition in the normal direction evaluated at the front and rear caps of the droplet,
Eq. (40), the pressure jump at the front and rear caps can also be computed as

$\Delta p_{f, r}=\gamma \chi \kappa_{f, r}+\Delta \tau_{z z_{f, r}}$.

Thus, with the proposed models (41) and (44) for the interface curvatures and normal viscous stress jumps at hand, the pressure jump model reads

$$
\begin{aligned}
\frac{\Delta p_{f, r} R}{\gamma}= & \frac{M_{f, r}(\lambda)(3 C a)+N_{f, r}(\lambda)(3 C a)^{4 / 3}}{1+O_{f, r}(\lambda)(3 C a)} \\
& +\chi \frac{1+T_{f, r}(\lambda)(3 C a)^{2 / 3}}{1+Z_{f, r}(\lambda)(3 C a)^{2 / 3}},
\end{aligned}
$$

which agrees with the FEM-ALE data better than Eq. (43) does (see dashed lines on Figs. 22 and 23 or Figs. 29 and 30p. Therefore, the jump in normal viscous stresses has to be taken into account for $\mathrm{Ca}>10^{-3}$.

\section{Stresses distribution and total pressure drop}

\subsection{Stresses distribution along the channel centerline}

The flow field can be classified into parallel and non-parallel regions (see Fig. 8). The parallel regions compose of the region sufficiently far away from the droplet and that encompassing the uniform lubrication film of constant thickness, where the flow is streamwise invariant. The profile of the streamwise velocity $u(r)$ is parabolic and the radial velocity $v \approx 0$. On the contrary, the flow is not parallel near the droplet extremities (see Fig. 8) where the flow stagnates. Therefore the nearby streamwise velocity vary significantly, leading to a non-zero radial velocity $v$ owing to the divergencefree condition.

We show in Fig. 24 the distribution of the total stress component $\sigma_{z z}=-p+\tau_{z z}$, of the pressure $p$ and of the viscous stress component $\tau_{z z}=2 \mu \partial u / \partial z$ along the centerline of the channel. $\tau_{z z}$ vanishes where the flow is approximately parallel. As seen in Sec. 7.3, $\tau_{z z}$ is negligible at small $C a$, typically below $10^{-3}$.

Furthermore, for a larger but still moderate $C a$ number, it is observed in Fig. 24(b) that the pressure (red line) deviates from the linearly varying pressure, $p_{\text {linear }}$ (black line), of the unperturbed flow (without droplet) featured with a constant pressure gradient. The deviation is attributed to the non-parallel flow structure near the front and rear caps of the droplet (see Fig. 8), hence the pressure based on $p_{\text {linear }}$ need to be corrected by $\Delta p^{\mathrm{NP}}=p-p_{\text {linear }}$. Typical values for the pressure corrections can be found in the Appendix $G$ These corrections are particularly large at large viscosity ratios for the region inside of the droplet. We did not succeed in providing a model to quantify this pressure correction.

Finally, in agreement with the results of Section 7, the jump in total stress or pressure at the rear of the droplet is smaller than the one at the front. 


\subsection{Pressure distribution along the channel wall}

The pressure distribution on the channel wall is presented on Fig. 24 as well (continuous grey line). The influence of the interface mean curvature is clearly visible. The non-monotonic pressure at the wall close the droplet rear results from the variation of the plane curvature in the dynamic meniscus region, where the interface oscillates (see also Fig. 11).

\subsection{Droplet-induced total pressure drop along a channel}

The prediction of the total pressure drop along a channel induced by the presence of a droplet flowing with a velocity $U_{d}$ is of paramount importance for the design of two-phase flow pipe networks [6, 37]. This allows for a coarse-grained quantification of the complicated local effects induced by the droplet. Droplets can thus be seen as punctual perturbations in the otherwise linear pressure evolution. In this section, we will show that it is possible to predict the total pressure drop induced by a droplet with the models proposed so far.

The total pressure drop can be defined as the difference between the pressure in the outer phase ahead and behind the droplet, namely $\Delta p_{\text {tot }}=p_{f}^{o}-p_{r}^{o}$ [35]. It is given by

$$
\begin{aligned}
\Delta p_{\text {tot }} & =\Delta p_{o, r}^{\mathrm{NP}}+\Delta p_{r}-\Delta p_{i, r}^{\mathrm{NP}} \\
& +\frac{d p_{i}}{d z} L_{d}+\Delta p_{i, f}^{\mathrm{NP}}-\Delta p_{f}-\Delta p_{o, f}^{\mathrm{NP}},
\end{aligned}
$$

where $\Delta p_{f, r}$ are given by the model for the pressure jumps at interfaces, equation (46). The pressure gradient $d p_{i} / d z$ in the parallel region inside the droplet is given by Eq. (31) and Eq. 67) for the axisymmetric and planar geometries, respectively. Assuming the droplet of volume/area $\Omega$ (axisymmetric/planar geometry) as a composition of two hemispherical caps of radius $R-H_{\infty}$, with $H_{\infty}$ given by Eq. [30, connected by a cylinder of the same radius, the droplet length $L_{d}$ can be approximated at first order for low $\mathrm{Ca}$, i.e. for $H_{\infty} / R \ll 1$, as

$L_{d}=\frac{\left(R+2 H_{\infty}\right)}{\pi} \frac{\Omega}{R^{3}}+\frac{2}{3}\left(R-H_{\infty}\right)$

for the axisymmetric case and

$L_{d}=\frac{\left(R+H_{\infty}\right)}{2} \frac{\Omega}{R^{2}}+\frac{4-\pi}{2}\left(R-H_{\infty}\right)$

for the planar case.

Equivalently, the total pressure drop can also be calculated using the models for the normal viscous stress jump, equation (44), and the front and rear plane curvatures, equation 41, yielding:

$$
\begin{aligned}
\Delta p_{\text {tot }} & =\Delta p_{o, r}^{\mathrm{NP}}+\Delta \tau_{z z_{r}}+\chi \gamma \kappa_{r}-\Delta p_{i, r}^{\mathrm{NP}} \\
& +\frac{d p_{i}}{d z} L_{d}+\Delta p_{i, f}^{\mathrm{NP}}-\Delta \tau_{z z_{f}}-\chi \gamma \kappa_{f}-\Delta p_{o, f}^{\mathrm{NP}},
\end{aligned}
$$

where $\chi=2$ for the axisymmetric configuration and $\chi=1$ for the planar one.

If we neglect the non-parallel flows effects on the pressure, $\Delta p^{\mathrm{NP}}$, the total pressure drop would then be:

$\Delta p_{\text {tot }}=\Delta p_{r}+\frac{d p_{i}}{d z} L_{d}-\Delta p_{f}$

Neglecting the effects of the non-parallel flows would induce an error on the pressure drop, increasing with $\mathrm{Ca}$. For a single droplet of volume $\Omega=12.9$, the error of Eq. (51) compared to the numerical results is less than $3 \%$ for $\lambda=0$, but reaches $15 \%$ for $\lambda=1$ and even $48 \%$ for $\lambda=100$. It is thus important to include the corrections accounting for the non-parallel flow effects to predict the pressure drop accurately, especially when the viscosity ratios $\lambda \gtrsim 1$. Numerical simulations are therefore crucial to achieve so.

\section{Conclusions}

This paper generalizes the theory of a confined bubble flowing in an axisymmetric or planar channel to droplets of nonvanishing viscosity ratios. Empirical models for the relevant quantities such as the uniform and minimal film thicknesses separating the wall and the droplet, the front and rear droplet plane curvatures, the total pressure drop in the channel and the droplet velocity are derived for the range of capillary numbers from $10^{-4}$ to 1 , and viscosity ratios ranging from the value $\lambda=0$ for bubbles to highly-viscous droplets. Following the work of Schwartz et al. [52], we extend the lowcapillary-number predictions obtained by the lubrication approach of Bretherton [9] for bubbles to viscous droplets. Extensive accurate moving-mesh arbitrary Lagrangian-Eulerian (ALE) finite-element numerical simulations are performed for the viscosity-ratio range $\lambda \in[0-100]$ to build a numerical database, based on which we propose empirical models for the relevant quantities. The models are inspired by the low- $\mathrm{Ca}$ theoretical asymptotes, but their validity range reaches large capillary numbers $\left(\mathrm{Ca}>10^{-3}\right)$, where the lubrication approach no longer holds.

We have found that the uniform film thickness for $\mathrm{Ca}<$ $10^{-3}$ does not differ significantly with that of a bubble as long as $\lambda<1$. For larger viscosity ratios, instead, the film thickness increases monotonically and saturates to a value $2^{2 / 3}$ times the Bretherton's scaling for bubbles when $\lambda>$ $10^{3}$. The film thickness can be modeled by a rational function similar to that proposed by Aussillous and Quéré [5] for bubbles, where the fitting coefficient $Q$ depends on the viscosity ratio. Furthermore, the uniform film thickness saturates at large capillary numbers to a value depending on $Q$. The minimum film thickness can be predicted analogously. The velocity of a droplet can be unambiguously derived once the uniform film thickness is known. We have shown that 
considering the full expression of the droplet velocity is crucial as the asymptotic series for low $\mathrm{Ca}$ has a very restricted range of validity for non-vanishing viscosity ratios.

Furthermore, we have found that the evolution of the front and rear cap curvatures as a function of the capillary number differs from the one of the pressure jumps at the front and rear droplet interfaces. This is due to the normal viscous stress jumps. The contribution of the jumps has been overlooked in the literature, though it has to be considered for $C a>10^{-3}$. With all these models at hand, the pressure drop across a droplet can be computed, which will be valuable for engineering practices.

We also have shown that the flow patterns inside and outside of the droplet strongly depend on the capillary number and viscosity ratio. In particular, for $\lambda<1 / 2(\lambda<2 / 3)$ for the axisymmetric (planar) configuration, when the film thickness is larger than a critical value $H_{\infty}^{\star} / R$, recirculating regions at the front and rear of the droplet disappear. Furthermore, the recirculation region in the outer phase detaches from the droplet's rear interface for large film thickness yet smaller than $H_{\infty}^{\star} / R$, implying the disappearance of the inner recirculating region at the rear.

The considered problem in a planar configuration could be relevant for the study of a front propagation in a HeleShaw cell [46, 48], where the second-phase viscosity is nonvanishing. For instance, one could compute the amount of fluid left on the walls when a finger of immiscible fluid penetrates [51]. Furthermore, the problem in the planar configuration can be seen as a first step towards understanding the dynamics of pancake droplets in a Hele-Shaw cell [29,61]. Another possible outlook is the extension of the present theory to capillaries with polygonal cross sections, where the film between the droplet and the walls is not axisymmetric, but thick films known as gutters develop in the capillary corners. Three-dimensional numerical simulations are then necessary to resolve this asymmetry. A force balance will determine the droplet velocity and an equivalent pressure drop model could be proposed for these geometries.

Despite the fact that this work was motivated by the vast number of droplet-based microfluidic applications, the analytically derived equation (24) serves as a generalization of the well known Landau-Levich-Derjaguin-Bretherton equation [38, 13,9] when the second fluid has a non-negligible viscosity. This equation could therefore be adapted to predict the film thickness in coating problems with two immiscible liquids.

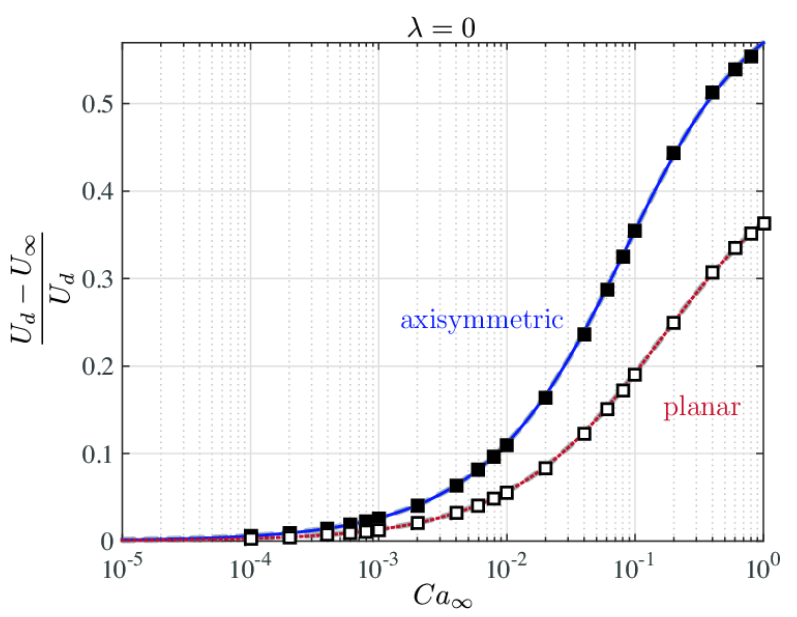

(a)

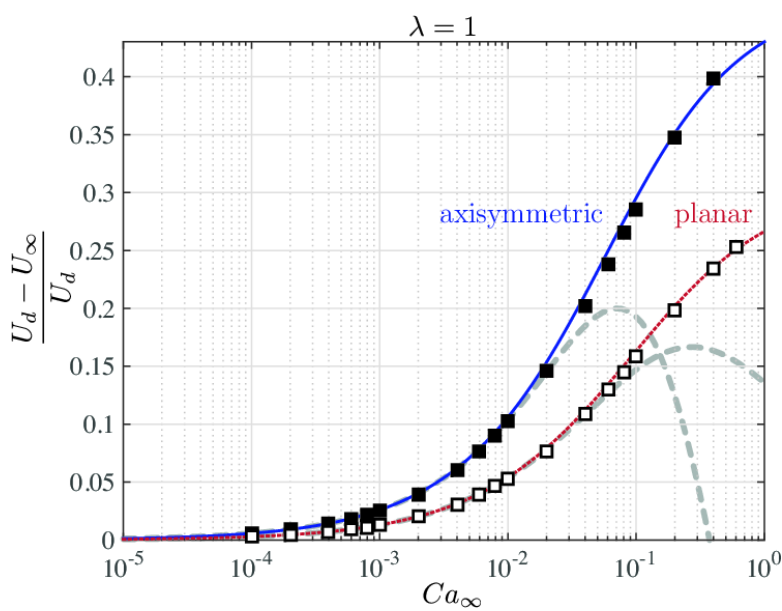

(b)

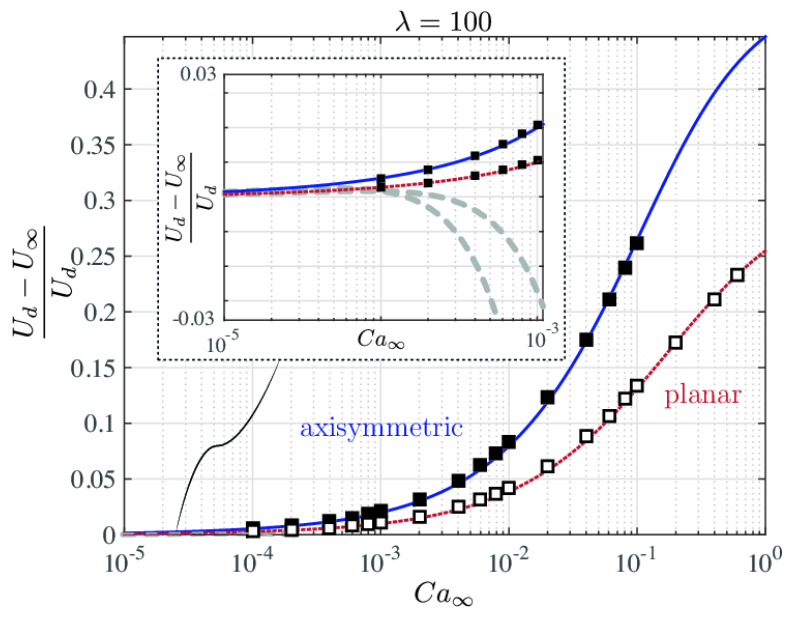

(c)

Fig. 16 Relative droplet velocity (lines) predicted by Eqs. (33) and (34) together with the proposed empirical model for the uniform film thickness $(30)$ and the results of the FEM-ALE numerical simulations (symbols) as a function of capillary number $C a_{\infty}$ for $\lambda=0$ (a), 1 (b) and 100 (c) and both axisymmetric (blue solid line, full symbols) and planar (dashed red line, empty symbols) geometries. Long dashed gray lines correspond to the asymptotic estimates of Eqs. 35. and (36). 


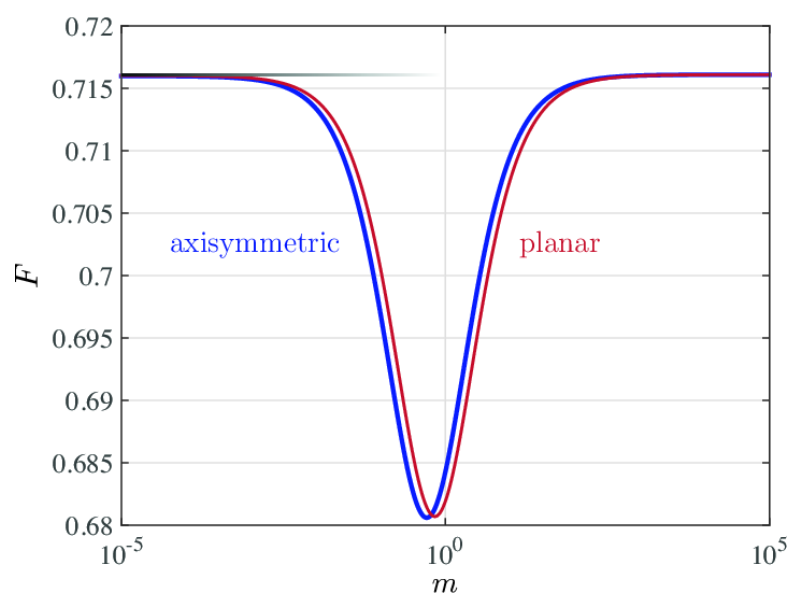

(a)

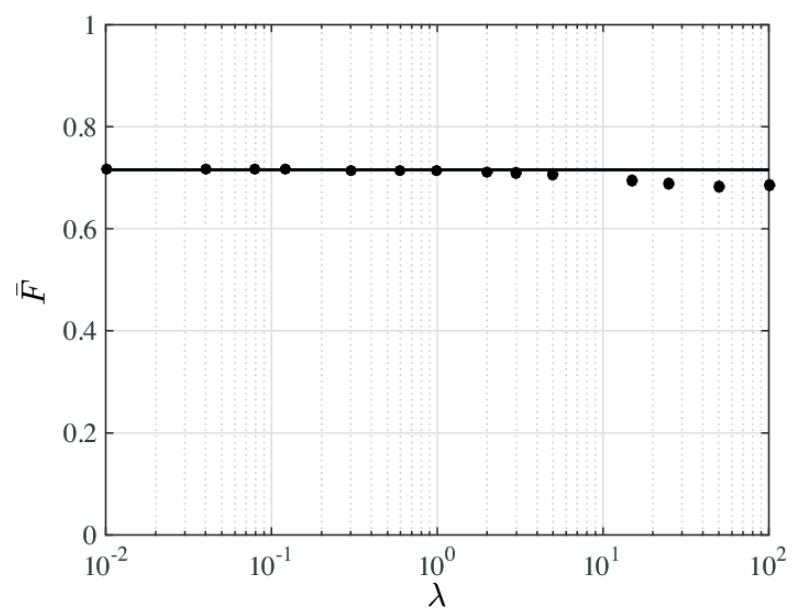

(b)

Fig. 17 (a) Minimum film-thickness coefficient $F$ as a function of the rescaled viscosity ratio $m$. (b) Mean coefficient $\bar{F}$ (symbols) and fitting law (solid line) as a function of the viscosity ratio $\lambda$

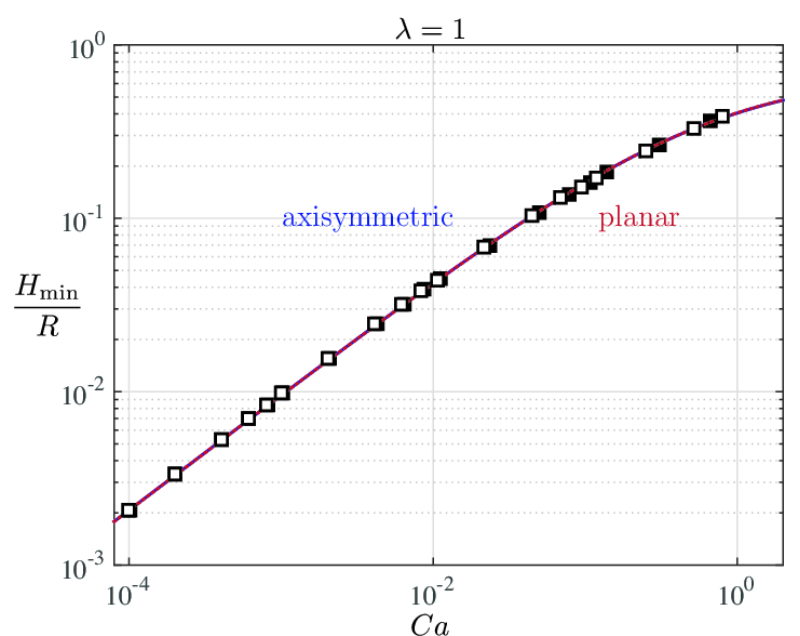

(a)

Fig. 18 Minimum film thickness given by Eq. 38) (lines) and FEMALE numerical results (symbols) as a function of the droplet capillary number for $\lambda=1$ and both axisymmetric (blue solid line, full symbols) and planar (dashed red line, empty symbols) geometries.

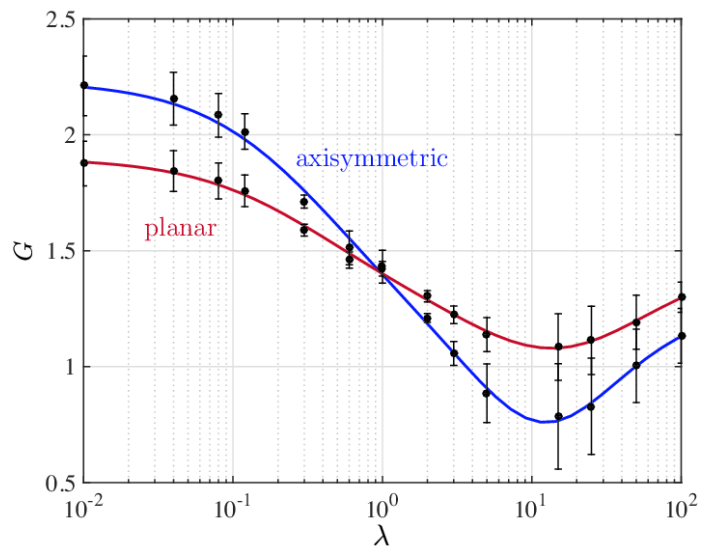

Fig. 19 Coefficient $G$ obtained for the simulated viscosity ratios (symbols) and proposed fitting law (71) (solid lines) as a function of the viscosity ratio $\lambda$. 


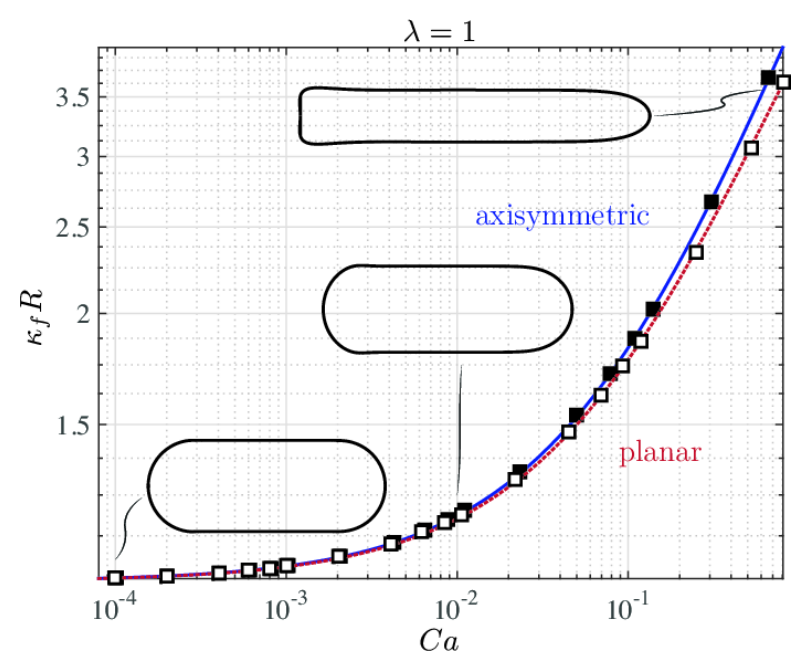

(a)

Fig. 20 Curvature $\kappa_{f}$ of the front meniscus predicted by the model Eq. (41) (lines) and FEM-ALE data (symbols) versus $\mathrm{Ca}$ for both axisymmetric (blue line, full symbols) and planar (red dashed line, empty symbols) geometries, where the viscosity ratio $\lambda=1$.

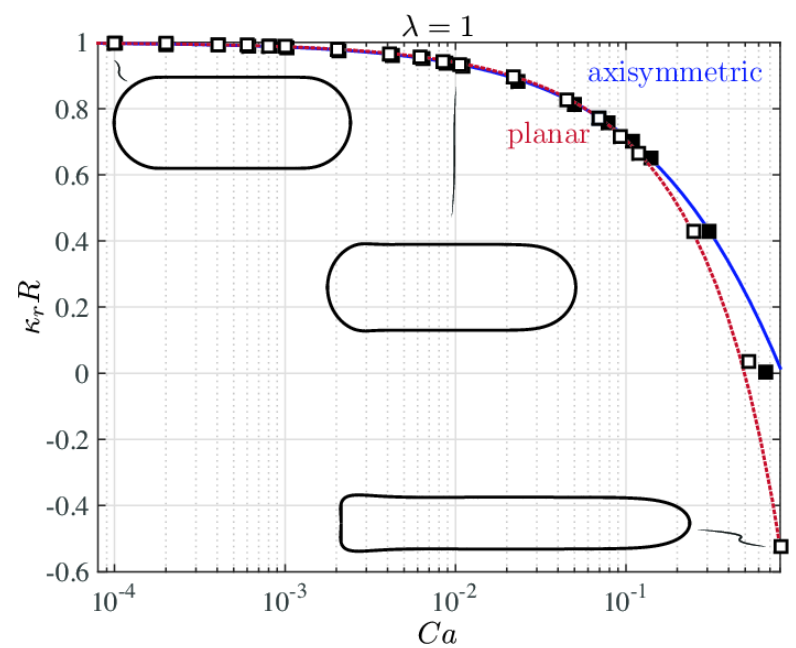

(a)

Fig. 21 The rear counterpart $\kappa_{r}$ of Fig. 20

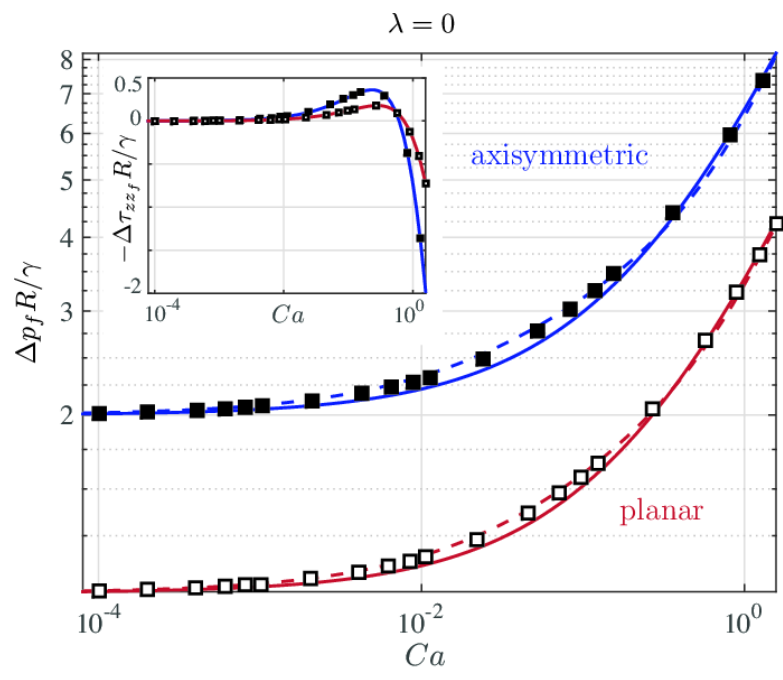

(a)

Fig. 22 Front pressure jump $\Delta p_{f}$ given by Eq. (43) (solid lines) and front normal viscous stress jump $\Delta \tau_{z z f}$ by Eq. 44) (inset, solid lines) and FEM-ALE data (symbols) versus $\mathrm{Ca}$ for both axisymmetric (blue line, full symbols) and planar (red line, empty symbols) geometries, where the viscosity ratio $\lambda=0$. The dashed lines correspond to the improved pressure jump model Eq. 46. Note the different scale in the insets.

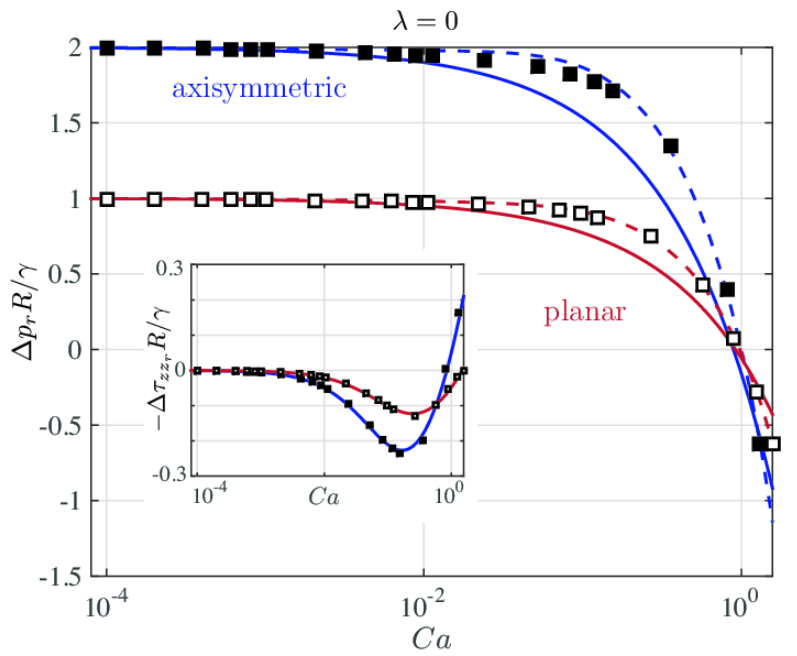

(a)

Fig. 23 The rear counterpart, pressure jump $\Delta p_{r}$ and normal viscous stress jump $\Delta \tau_{z z_{r}}$, of Fig. 22 


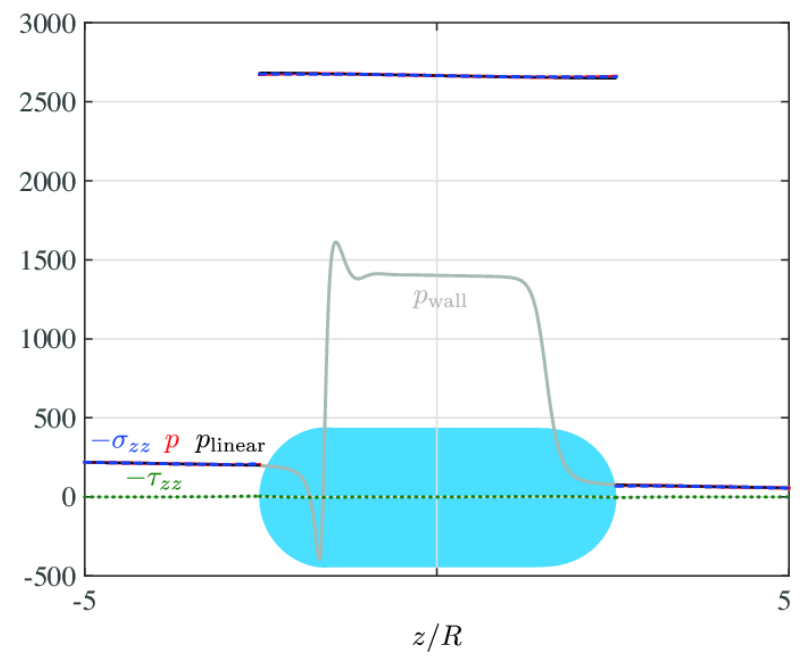

(a)

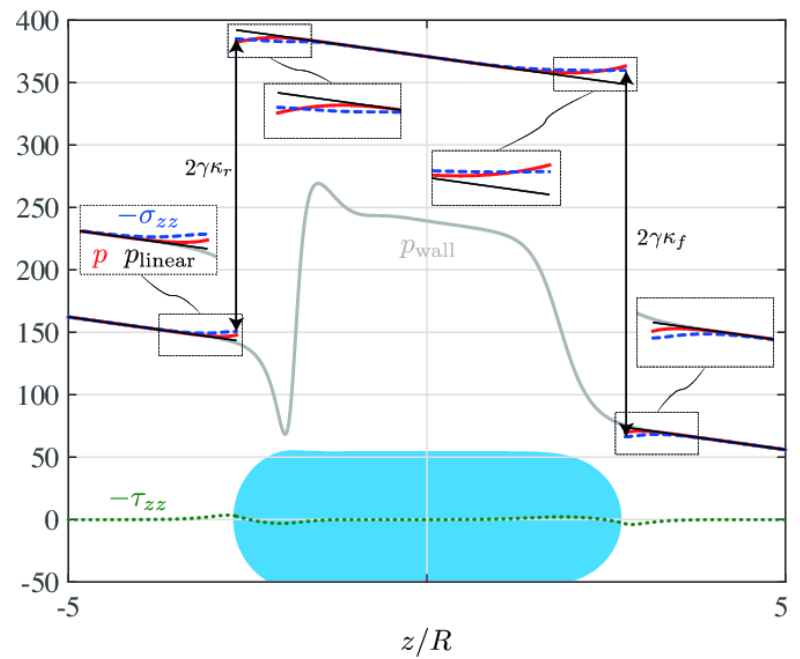

(b)

Fig. 24 Spatial evolution of the pressure $p$ (red solid line), normal viscous stresses $-\tau_{z z}$ (green dotted line) and total stresses $-\sigma_{z z}$ (blue dashed line) along the centerline for $C a=8.2 \cdot 10^{-4}$ (a) and $C a=$ $8.8 \cdot 10^{-3}(\mathrm{~b}), \lambda=1$ and an axisymmetric configuration, obtained from the FEM-ALE numerical simulations. The linear pressure evolution without considering non-parallel flow effects is shown by the thin black lines. The total stresses jumps induced by the mean curvature at the interfaces are indicated by arrows. The droplet shape is indicated in blue. The pressure at the channel wall is indicated by the grey line. 


\section{A Derivation of the flow profiles in the thin-film region for the planar configuration}

Consider an axial location in the thin-film region. The velocity profiles inside, $u_{i}$, and outside, $u_{o}$, of the droplet can be described by:

$u_{i}(r)=\frac{1}{2 \mu_{i}} \frac{d p_{i}}{d z} r^{2}+A_{i} r+B_{i}$,
$u_{o}(r)=\frac{1}{2 \mu_{o}} \frac{d p_{o}}{d z} r^{2}+A_{o} r+B_{o}$,

where $p_{i}$ and $p_{o}$ are the inner, respectively outer, pressures, and $A_{i}, B_{i}$, $A_{o}$ and $B_{o}$ are real constants to be determined. Given the symmetry at $r=0$ of the inner velocity, $A_{i}=0$. The other constants are found by imposing the no-slip boundary condition at the channel walls $u(R)=-U_{d}$ in the droplet reference frame, the continuity of velocities at the interface located at $r=R-H, u_{i}(R-H)=u_{o}(R-H)$, and the continuity of tangential stresses at the interface

$\left.\mu_{i} \frac{d u_{i}}{d z}\right|_{r=R-H}=\left.\mu_{o} \frac{d u_{o}}{d z}\right|_{r=R-H}$.

Eventually one obtains:

$A_{o}=\frac{1}{\mu_{o}}\left(\frac{d p_{i}}{d z}-\frac{d p_{o}}{d z}\right)(R-H)$,

$B_{i}=\frac{1}{2 \mu_{i} \mu_{o}}\left[-(R-H)^{2} \frac{d p_{i}}{d z} \mu_{o}+H\left(2 H \frac{d p_{i}}{d z}-H \frac{d p_{o}}{d z}\right.\right.$

$\left.\left.-2 R \frac{d p_{i}}{d z}\right) \mu_{i}\right]-U_{d}$

$B_{o}=\frac{1}{2 \mu_{o}}\left[\left(\frac{d p_{o}}{d z}-2 \frac{d p_{i}}{d z}\right) R^{2}-2 H R\left(\frac{d p_{o}}{d z}-\frac{d p_{i}}{d z}\right)\right]-U_{d}$.

\section{B Derivation of the interface profile equation for the planar configuration}

The flow rates at any axial location where the external film thickness is $H$ are:

$$
\begin{aligned}
q_{i} & =2 \int_{0}^{R-H} u_{i}(r) d r \\
& =\frac{1}{3 \mu_{i} \mu_{o}}\left\{-(R-H)\left[3 H\left(H\left(\frac{d p_{o}}{d z}-2 \frac{d p_{i}}{d z}\right)+2 \frac{d p_{i}}{d z} R\right) \mu_{i}\right.\right. \\
& \left.\left.+2 \frac{d p_{i}}{d z}(R-H)^{2} \mu_{o}\right]\right\}-2 U_{d}(R-H), \\
q_{o} & =2 \int_{R-H}^{R} u_{o}(r) d r \\
& =\frac{H^{2}}{3 \mu_{o}}\left[H\left(3 \frac{d p_{i}}{d z}-2 \frac{d p_{o}}{d z}\right)-3 \frac{d p_{i}}{d z} R\right]-2 U_{d} H .
\end{aligned}
$$

In the droplet reference frame, the flow rate of the inner phase has to vanish, $q_{i}=0$. Furthermore, in the region where the film is uniform (see Fig. 11, $H=H_{\infty}$, the inner and outer pressure gradients have to be equal. Using these two conditions one can solve for the pressure gradient in the uniform film region

$$
\left.\frac{d p}{d z}\right|_{r=R-H_{\infty}} \approx-\frac{6 \mu_{i} U_{d}}{2 R^{2}-(4-6 \lambda) H_{\infty} R+(2-3 \lambda) H_{\infty}^{2}}
$$

and for the outer flow rate, where the limit $H_{\infty} / R \ll 1$ is considered:

$$
\begin{aligned}
q_{o} & \approx-2 H_{\infty}\left[\frac{2 R^{2}-(4-3 \lambda) H_{\infty} R+2(1-\lambda) H_{\infty}^{2}}{2 R^{2}-(4-6 \lambda) H_{\infty} R+(2-3 \lambda) H_{\infty}^{2}}\right] U_{d} \\
& \approx-H_{\infty}\left[\frac{2 R-(4-3 \lambda) H_{\infty}}{R-(2-3 \lambda) H_{\infty}}\right] U_{d}
\end{aligned}
$$

The pressure gradients in the dynamic meniscus regions are no longer equal and their difference is proportional to the deformation of the interface $r=R-H$. Under the assumption of a quasi-parallel flow, and neglecting the viscous contribution in view of the lubrication assumption, the Laplace law imposes:

$$
\frac{d p_{i}}{d z}-\frac{d p_{o}}{d z}=\gamma \frac{d^{3} H}{d z^{3}}
$$

Knowing $q_{i}$ and $q_{o}$, Eqs. 58, 59] can be solved for the unknown pressure gradients $d p_{i} / d z, d p_{o} / d z$ as a function of $H$ :

$$
\begin{aligned}
\frac{d p_{i}}{d z} \approx & \frac{3 \lambda\left\{2 H\left[H_{\infty}(3 \lambda-2)+R\right]-3 H_{\infty}\left[H_{\infty}(3 \lambda-4)+2 R\right]\right\} \mu_{o} U_{d}}{H(R-H)[H(3 \lambda-4)+4 R]\left[H_{\infty}(3 \lambda-2)+R\right]}, \\
\frac{d p_{o}}{d z} \approx & -6\left\{\frac{R\left(H-H_{\infty}\right)\left[3 \lambda\left(H+H_{\infty}\right)-2\left(H+2 H_{\infty}\right)\right]}{H^{3}[H(3 \lambda-4)+4 R]\left[H_{\infty}(3 \lambda-2)+R\right]}\right. \\
& \left.+\frac{H H_{\infty}\left[H(2-3 \lambda)^{2}+H_{\infty}(3(5-3 \lambda) \lambda-4)\right]+2 R^{2}\left(H-H_{\infty}\right)}{H^{3}[H(3 \lambda-4)+4 R]\left[H_{\infty}(3 \lambda-2)+R\right]}\right\} \mu_{o} U_{d}
\end{aligned}
$$

and substituted into Eq. (62). Following Bretherton [9], the resulting equation can be put in an universal form by the substitutions $H=H_{\infty} \eta$ and $z=H_{\infty}(3 \mathrm{Ca})^{-1 / 3} \xi$. In the limit of $H_{\infty} / R \rightarrow 0$, the governing equation for the interface profile reads:

$\frac{d^{3} \eta}{d \xi^{3}}=2 \frac{\eta-1}{\eta^{3}}\left[\frac{2+3 m(1+\eta+3 m \eta)}{(1+3 m)(4+3 m \eta)}\right]$.

where

$m=\lambda \frac{H_{\infty}}{R}$

is the rescaled viscosity ratio.

\section{Derivation of the droplet velocity model for the planar configuration}

The velocity profiles in the uniform film region have been derived in Appendix A In particular, the inner and outer volumetric fluxes are given by Eqs. 58) and (59), respectively. At the location where $H=$ $H_{\infty}$ the interface is flat and the pressure gradients are equal, $d p_{i} / d z=$ $d p_{o} / d z=d p / d z$. Furthermore, mass conservation imposes that $q_{o}=$ $2 R\left(U_{\infty}-U_{d}\right)$ and since we are in the reference frame of the droplet, $q_{i}=0$. The system of two equations can be solved for the pressure gradient

$$
\left.\frac{d p}{d z}\right|_{r=R-H_{\infty}}=\frac{-3 R U_{\infty} \mu_{i}}{\left(R-H_{\infty}\right)^{3}+H_{\infty}\left(3 R^{2}-3 H_{\infty} R+H_{\infty}^{2}\right) \lambda}
$$

and the droplet velocity

$U_{d}=\frac{R\left[2\left(R-H_{\infty}\right)^{2}+3 H_{\infty}\left(2 R-H_{\infty}\right) \lambda\right]}{2\left(R-H_{\infty}\right)^{3}+2 H_{\infty}\left(3 R^{2}-3 H_{\infty} R+H_{\infty}^{2}\right) \lambda} U_{\infty}$.

The relative velocity of the planar droplet reads

$\frac{U_{d}-U_{\infty}}{U_{d}}=\frac{\frac{H_{\infty}}{R}\left\{2-\frac{H_{\infty}}{R}\left[4+2 \frac{H_{\infty}}{R}(\lambda-1)-3 \lambda\right]\right\}}{2+\left(2-\frac{H_{\infty}}{R}\right) \frac{H_{\infty}}{R}(3 \lambda-2)}$. 
Table 1 Coefficients of the fitting law for the axisymmetric configuration.

\begin{tabular}{|c|c|c|c|c|}
\hline & $a_{0}$ & $a_{1}$ & $a_{2}$ & $a_{3}$ \\
\hline$Q$ & 2.21 & 111.25 & 33.84 & 1.37 \\
\hline$G$ & 130.37 & 186.67 & -4.82 & 1.30 \\
\hline$T_{f}$ & 3262.57 & 1573.07 & 7222.70 & 9.90 \\
\hline$T_{r}$ & -12031.57 & -21476.98 & 2820.73 & 77.21 \\
\hline$Z_{f}$ & 3392.32 & -1773.73 & 2984.79 & 39.56 \\
\hline$Z_{r}$ & -1842.14 & -14129.53 & 26169.48 & 160.45 \\
\hline$M_{f}$ & -4850.40 & 5797.90 & -507.02 & 1.22 \\
\hline$M_{r}$ & -6.38 & 18.59 & -10.85 & -0.82 \\
\hline$N_{f}$ & -5293.51 & 14808.02 & -9344.15 & -126.15 \\
\hline$N_{r}$ & -2.93 & -17.28 & 18.94 & 1.08 \\
\hline$O_{f}$ & 0.01 & -0.02 & 0.08 & -0.11 \\
\hline$O_{r}$ & 32.38 & -429.86 & 638.07 & -5.84 \\
\hline
\end{tabular}

\begin{tabular}{cccc}
\hline & $b_{0}$ & $b_{1}$ & $b_{2}$ \\
\hline$Q$ & 0.89 & 44.86 & 54.50 \\
$G$ & 58.41 & 154.37 & 10.56 \\
$T_{f}$ & 1197.26 & 2006.27 & 2855.96 \\
$T_{r}$ & 25461.62 & 11675.00 & 16374.62 \\
$Z_{f}$ & 5249.41 & 12649.53 & 32757.19 \\
$Z_{r}$ & 19514.41 & 14458.49 & 33771.45 \\
$M_{f}$ & 2412.12 & 2134.95 & -222.42 \\
$M_{r}$ & -2.68 & 4.97 & 2.93 \\
$N_{f}$ & -3171.89 & -3079.05 & 8185.38 \\
$N_{r}$ & 1.68 & 10.76 & 5.71 \\
$O_{f}$ & 0.06 & -0.06 & -0.64 \\
$O_{r}$ & 11.85 & -155.00 & 338.88 \\
\hline
\end{tabular}

\section{Derivation of the critical uniform film thickness for the appearance of the recirculation regions}

The velocity profile in the channel away from the droplet is given by Eq. (11) for the axisymmetric configuration and by

$u_{\infty}(y)=\frac{3}{2} U_{\infty}\left[1-\left(\frac{y}{R}\right)^{2}\right]-U_{d}$

for the planar one. The droplet velocity for the former case is given by Eq. (32), whereas for the latter it is given by Eq. 68). With the use of Eqs. (32) and (68), the velocity $u_{\infty}$ can be expressed as a function of $H_{\infty} / R$. The critical uniform film thickness for the appearance of recirculation regions, $H_{\infty}^{\star}$, is obtained by solving $u_{\infty}(0)=0$, resulting in Eqs. 12 and (13) for the axisymmetric and planar configurations, respectively.

\section{E Fitting laws for the model coefficients}

The model coefficients $Q$ in Eq. 30, $G$ in Eq. 38, $T_{f, r}$ and $Z_{f, r}$ in Eq. (41) and $M_{f, r}, N_{f, r}$ and $O_{f, r}$ in Eq. (44) can be well approximated by the rational function

$\frac{a_{3} \lambda^{3}+a_{2} \lambda^{2}+a_{1} \lambda+a_{0}}{\lambda^{3}+b_{2} \lambda^{2}+b_{1} \lambda+b_{0}}$,

where the constants $a_{i}$ with $i=0, . ., 3$ and $b_{j}$ with $j=0, . ., 2$ are given in tables 1 and 2 for the axisymmetric and planar geometries, respectively.
Table 2 Coefficients of the fitting law for the planar configuration.

\begin{tabular}{|c|c|c|c|c|}
\hline & $a_{0}$ & $a_{1}$ & $a_{2}$ & $a_{3}$ \\
\hline$Q$ & 98.76 & 146.42 & 70.42 & 1.45 \\
\hline$G$ & 168.27 & 348.60 & 26.76 & 1.48 \\
\hline$T_{f}$ & 0.35 & 1.17 & 5.41 & 2.43 \\
\hline$T_{r}$ & -130.18 & -298.84 & -55.49 & -0.66 \\
\hline$Z_{f}$ & 1096.45 & 191.51 & 395.61 & -0.08 \\
\hline$Z_{r}$ & -0.62 & -0.66 & 0.08 & -0.21 \\
\hline$M_{f}$ & -6.41 & 17.26 & -11.54 & 0.80 \\
\hline$M_{r}$ & 4.10 & -3.52 & 0.17 & 0.04 \\
\hline$N_{f}$ & -6.12 & 17.95 & -11.90 & 0.19 \\
\hline$N_{r}$ & -50.40 & 61.95 & -14.79 & 0.51 \\
\hline$O_{f}$ & 4.52 & -2.80 & -1.73 & 0.89 \\
\hline \multirow[t]{2}{*}{$O_{r}$} & 2.10 & -6.97 & 2.79 & -0.01 \\
\hline & $b_{0}$ & $b_{1}$ & $b_{2}$ & \\
\hline$Q$ & 45.04 & 89.61 & 77.03 & \\
\hline$G$ & 88.60 & 264.97 & 34.41 & \\
\hline$T_{f}$ & 0.16 & 0.57 & 3.02 & \\
\hline$T_{r}$ & 256.91 & 292.80 & 83.68 & \\
\hline$Z_{f}$ & 2690.72 & 6726.39 & 2249.29 & \\
\hline$Z_{r}$ & 5.80 & -0.74 & 3.08 & \\
\hline$M_{f}$ & 7.40 & -2.88 & -12.76 & \\
\hline$M_{r}$ & 4.55 & 7.67 & -5.25 & \\
\hline$N_{f}$ & -9.06 & -6.06 & 30.83 & \\
\hline$N_{r}$ & 84.57 & 41.55 & -24.20 & \\
\hline$O_{f}$ & 27.19 & -57.38 & 21.87 & \\
\hline$O_{r}$ & 1.14 & -1.38 & -1.96 & \\
\hline
\end{tabular}

\section{F Additional results}

For seek of clarity, the results for $\lambda=0$ and 100 are shown in the appendix rather than in the main text, except for the normal viscous stresses jump, whose results for $\lambda=0$ are presented in the main text as for $\lambda=1$ the normal viscous stress jumps are small.

\section{G Pressure corrections due to non-parallel flow}

Some typical total stresses corrections at the outer and inner sides of the droplet interface as a function of $\mathrm{Ca}$ and for different viscosity ratios $\lambda$ are shown in Fig. 31 and Fig. 32 respectively.

Acknowledgements This work was funded by ERC Grant No. 'SIMCOMICS 280117'. L.Z. gratefully acknowledges the VR International Postdoc Grant from Swedish Research Council '2015-06334' for financial support. The authors would like to acknowledge the valuable comments from the anonymous referees that helped to improve the manuscript. 


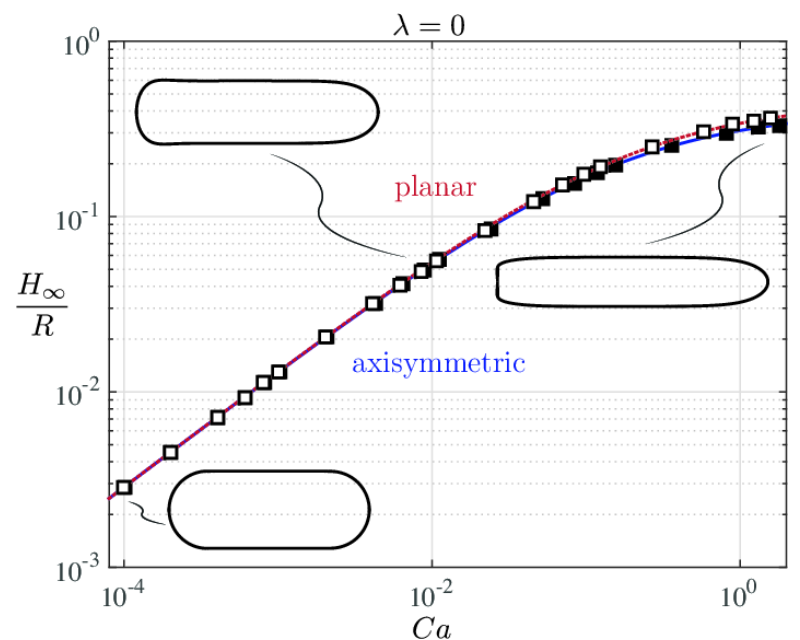

(a)

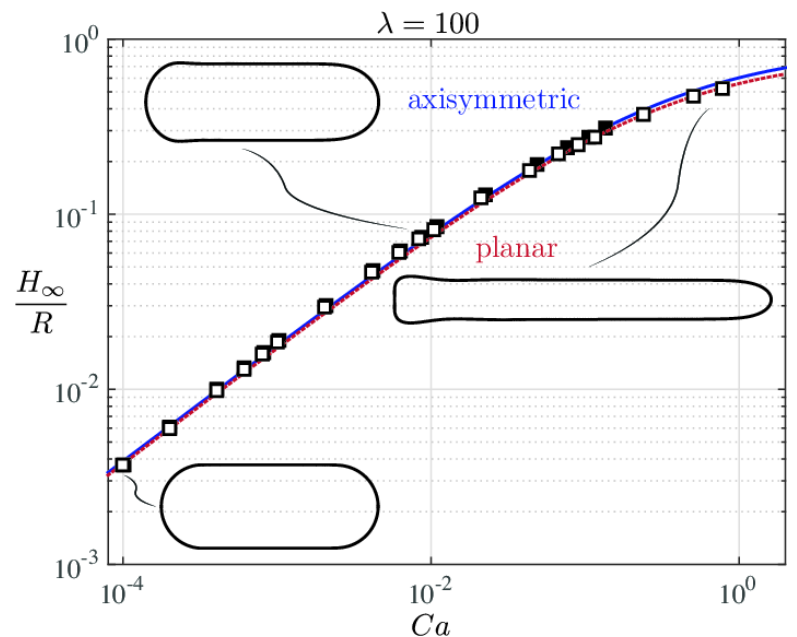

(b)

Fig. 25 Uniform film thickness given by Eq. (30) (lines) and FEMALE numerical results (symbols) as a function of the droplet capillary number for $\lambda=0$ (a) and 100 (b) and both axisymmetric (blue solid line, full symbols) and planar (dashed red line, empty symbols) geometries.

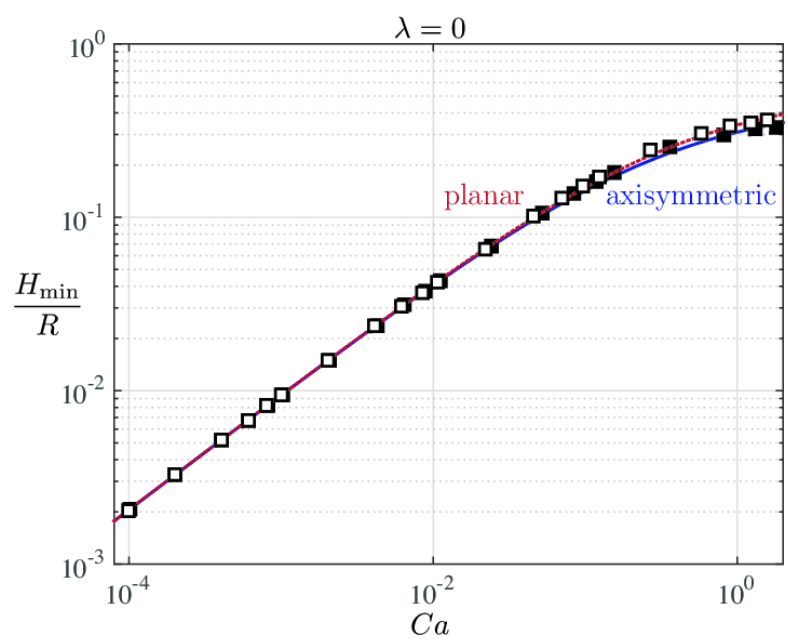

(a)

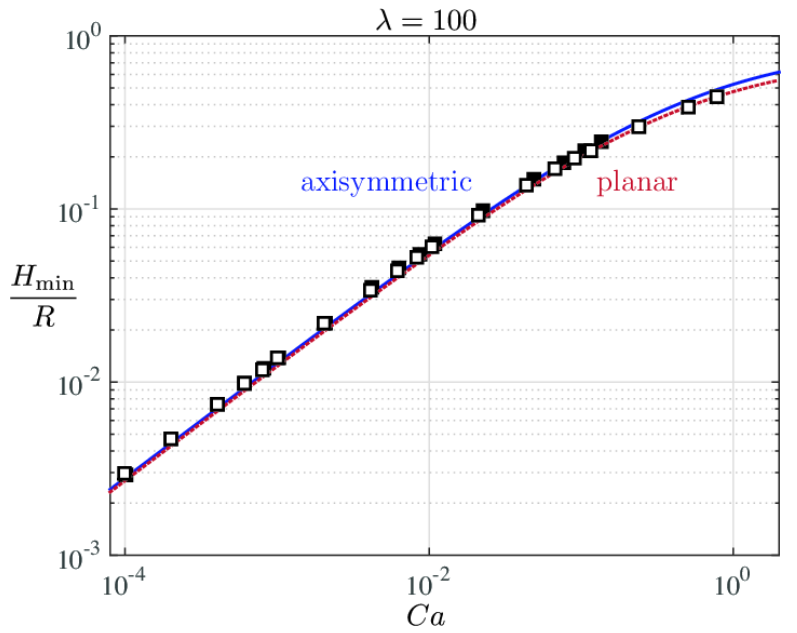

(b)

Fig. 26 Minimum film thickness given by Eq. (38) (lines) and FEMALE numerical results (symbols) as a function of the droplet capillary number for $\lambda=0$ (a) and 100 (b) and both axisymmetric (blue solid line, full symbols) and planar (dashed red line, empty symbols) geometries. 


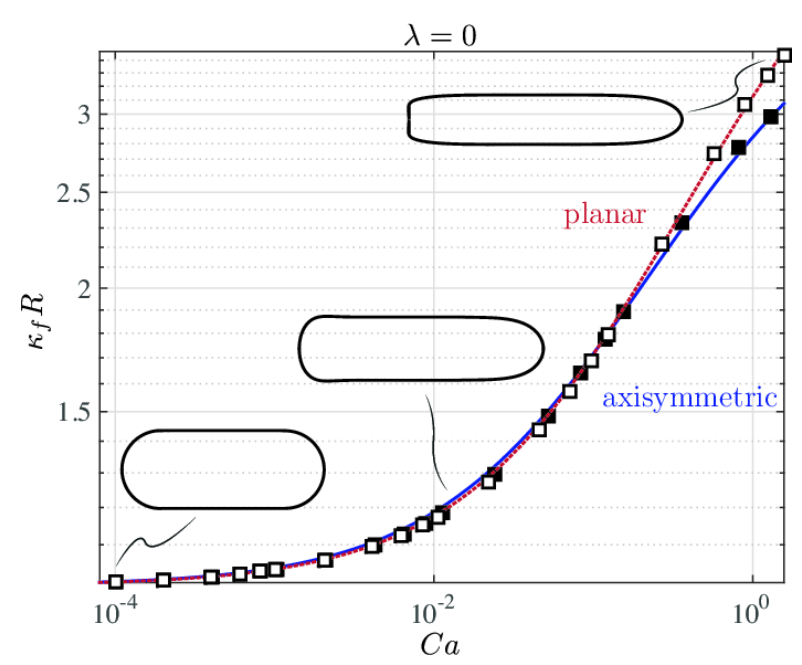

(a)

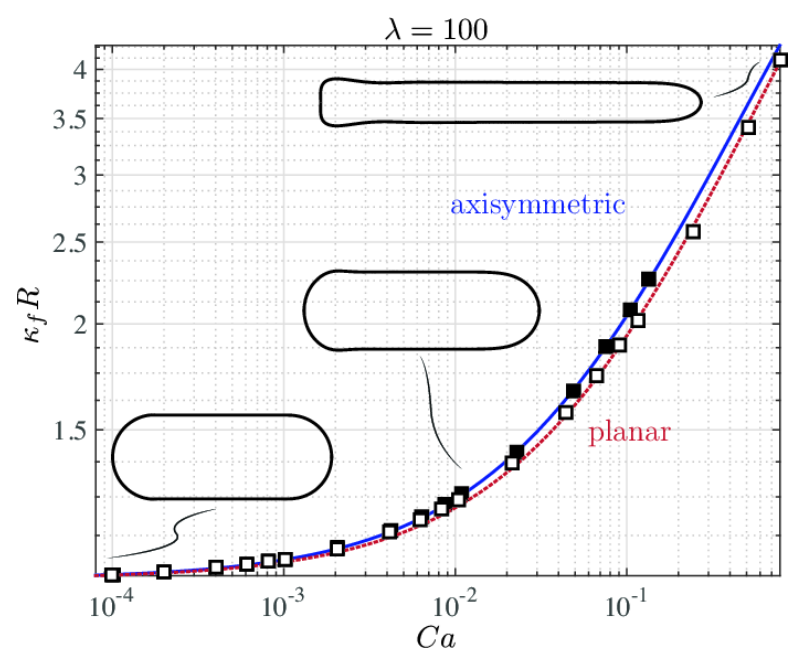

(b)

Fig. 27 Curvature $\kappa_{f}$ of the front meniscus predicted by the model Eq. (41) (lines) and FEM-ALE data (symbols) versus $\mathrm{Ca}$ for both axisymmetric (blue line, full symbols) and planar (red dashed line, empty symbols) geometries, where the viscosity ratio $\lambda=0$ (a) and 100 (b).

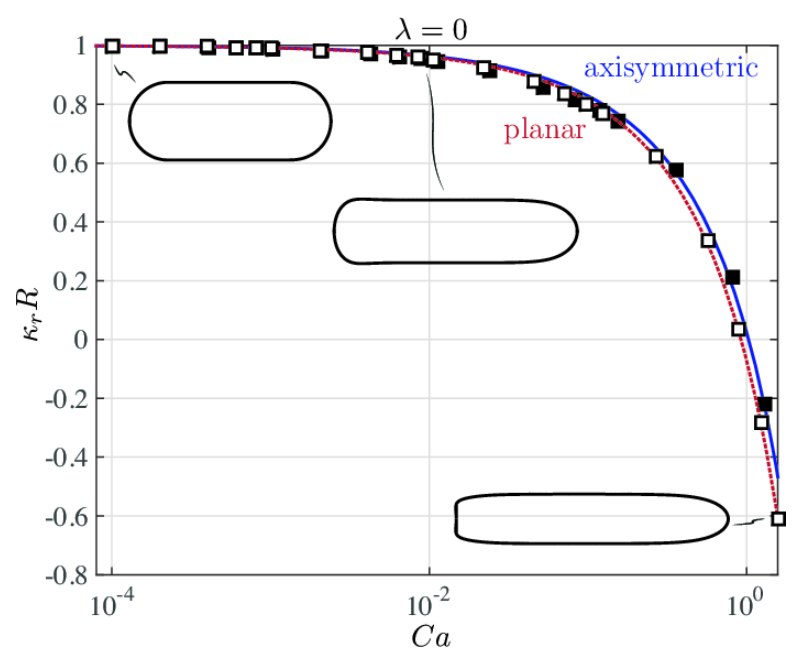

(a)

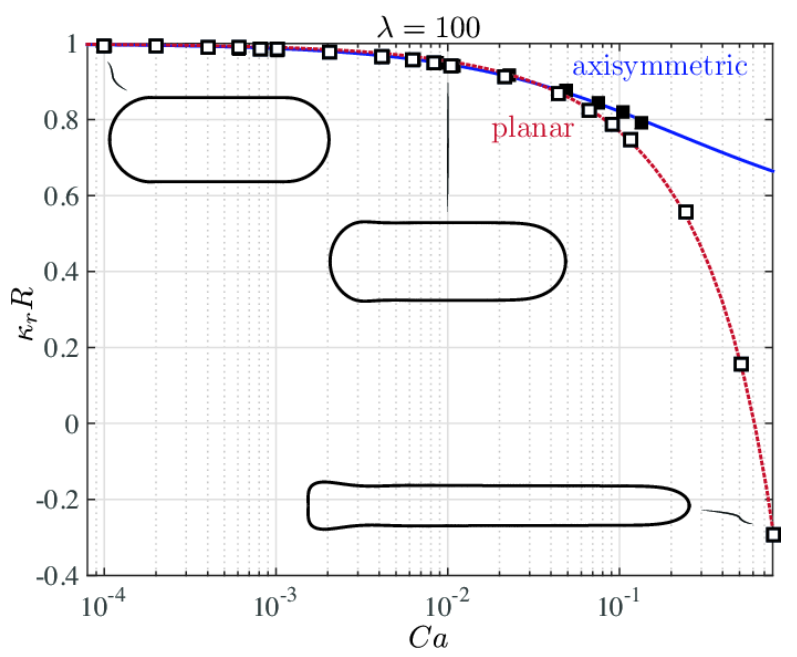

(b)

Fig. 28 The rear counterpart $\kappa_{r}$ of Fig. 27 


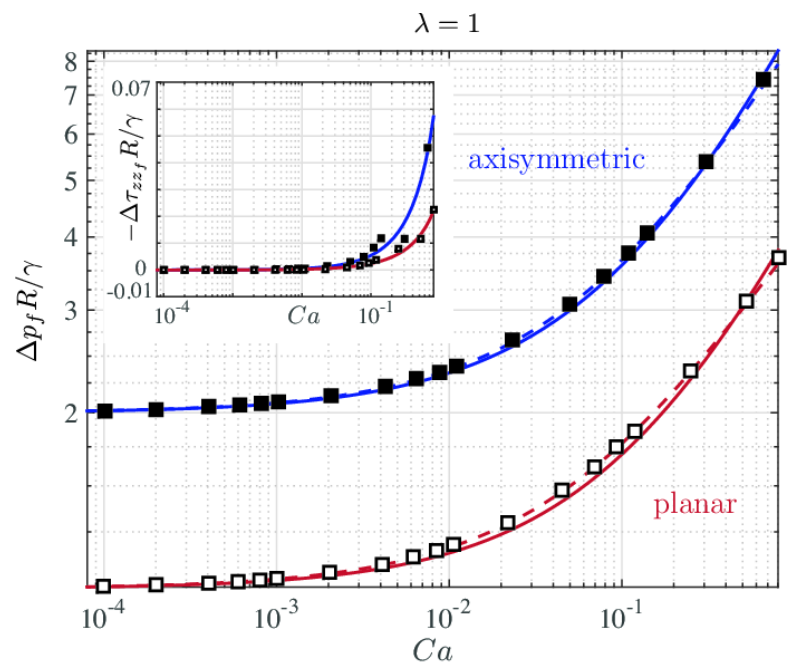

(a)

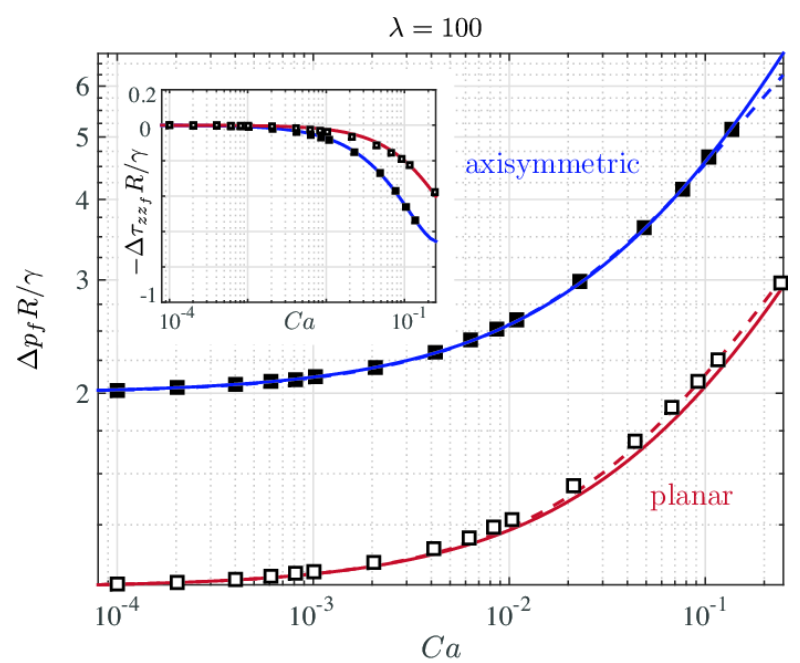

(b)

Fig. 29 Front pressure jump $\Delta p_{f}$ given by Eq. (43) (solid lines) and front normal viscous stress jump $\Delta \tau_{z z f}$ by Eq. 44f (inset, solid lines) and FEM-ALE data (symbols) versus $\mathrm{Ca}$ for both axisymmetric (blue line, full symbols) and planar (red line, empty symbols) geometries, where the viscosity ratio $\lambda=1$ (a) and 100 (b). The dashed lines correspond to the improved pressure jump model Eq. 46. Note the different scale in the insets.

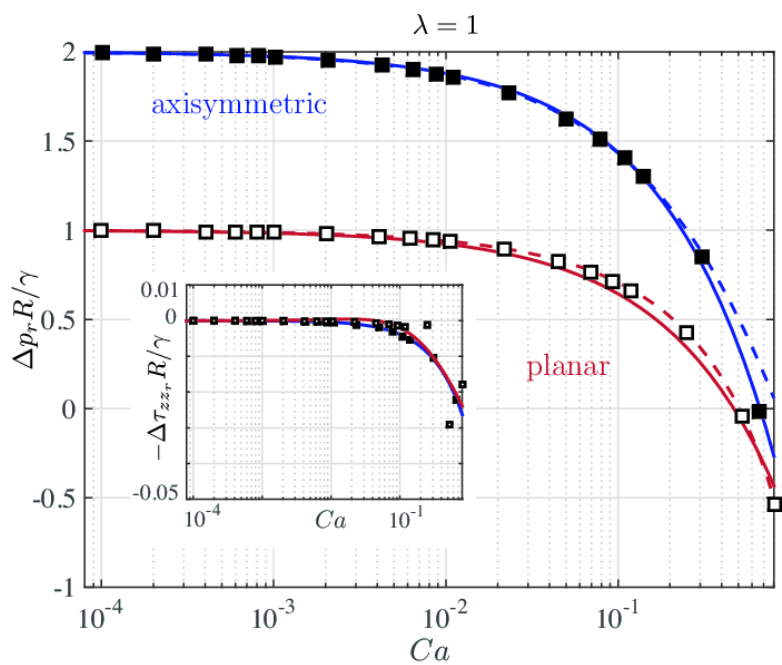

(a)

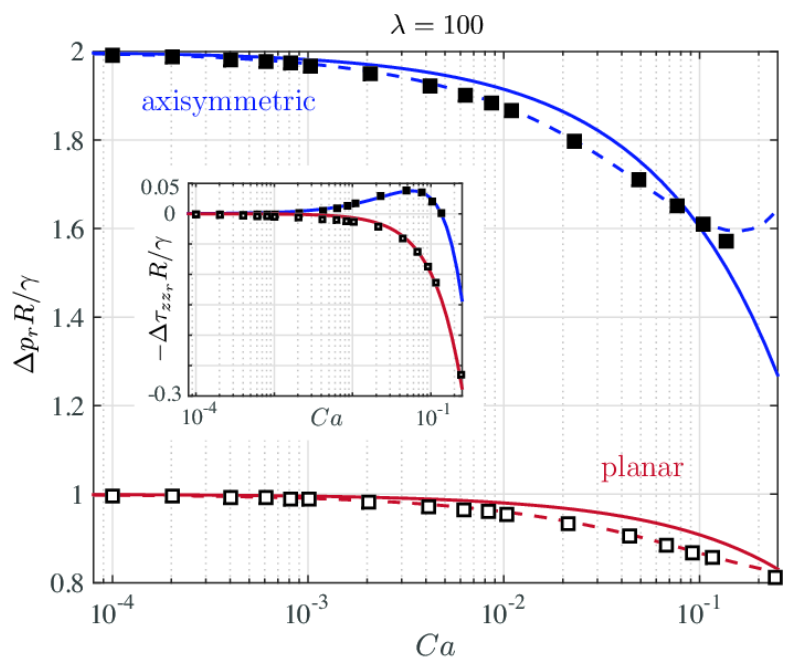

(b)

Fig. 30 The rear counterpart, pressure jump $\Delta p_{r}$ and normal viscous stress jump $\Delta \tau_{z z r}$, of Fig. 29 


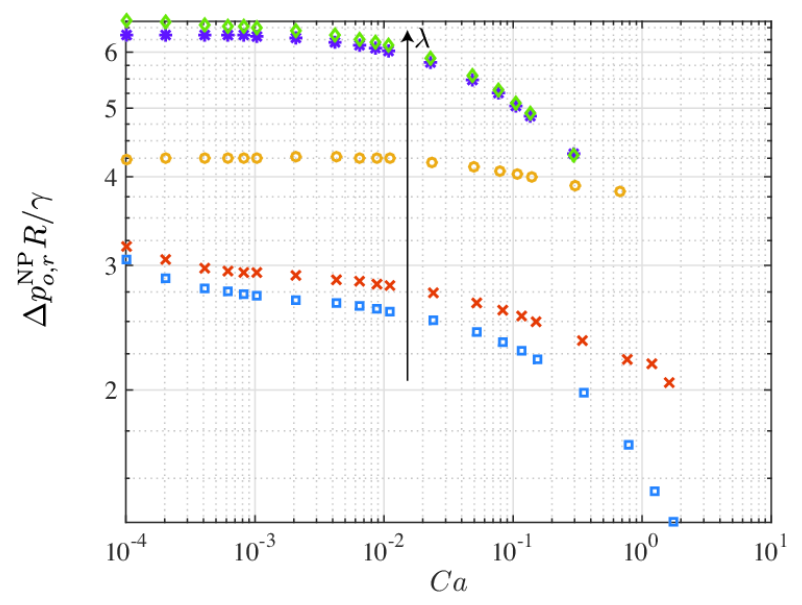

(a)

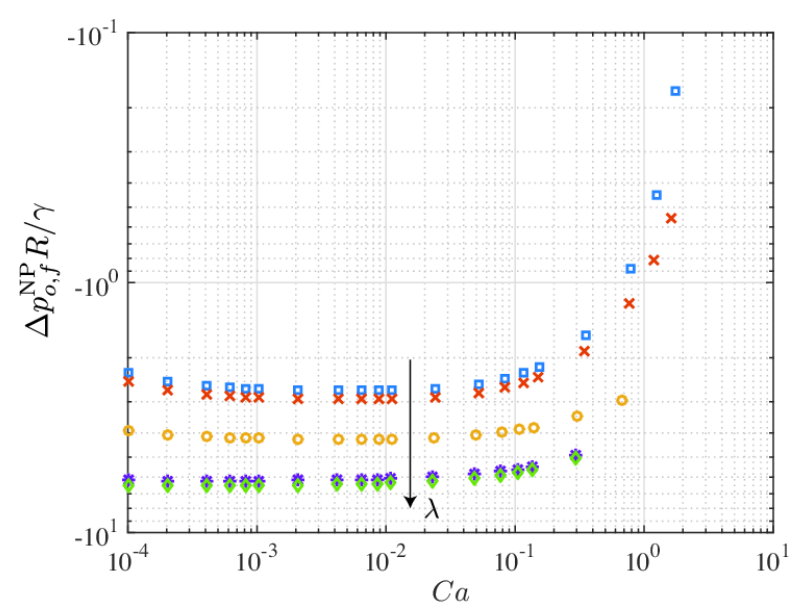

(b)

Fig. 31 Pressure correction due to non-parallel flow effects at the rear (a) and front (b) outer sides of the interface for $\lambda=0.04$ (blue squares), 0.12 (red crosses), 1 (yellow circles), 15 (purple stars) and 50 (green diamonds) for the axisymmetric configuration. The results are obtained from FEM-ALE numerical simulations.

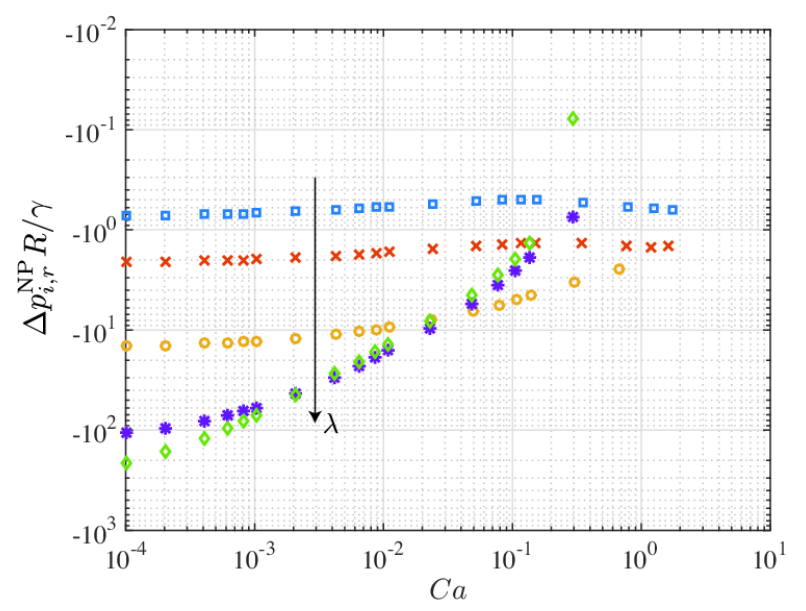

(a)

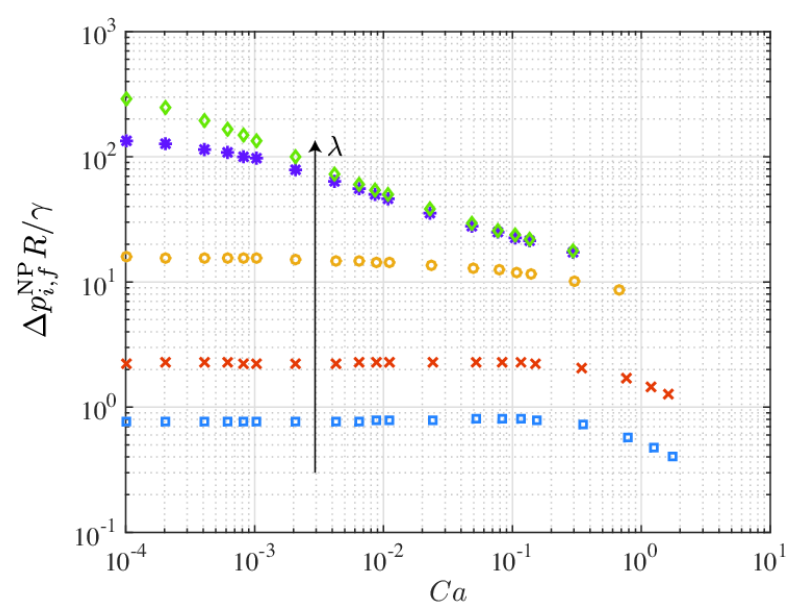

(b)

Fig. 32 Pressure correction due to non-parallel flow effects at the rear (a) and front (b) inner sides of the interface for $\lambda=0.04$ (blue squares), 0.12 (red crosses), 1 (yellow circles), 15 (purple stars) and 50 (green diamonds) for the axisymmetric configuration. The results are obtained from FEM-ALE numerical simulations. 


\section{References}

1. Abadie, T., Aubin, J., Legendre, D., Xuereb, C.: Hydrodynamics of gas-liquid Taylor flow in rectangular microchannels. Microfluidics and Nanofluidics 12(1-4), 355-369 (2012)

2. Anjos, G., Mangiavacchi, N., Borhani, N., Thome, J.R.: 3D ALE finite-element method for two-phase flows with phase change. Heat Transfer Engineering 35(5), 537-547 (2014)

3. Anjos, G.R., Borhani, N., Mangiavacchi, N., Thome, J.R.: A 3D moving mesh finite element method for two-phase flows. Journal of Computational Physics 270, 366-377 (2014)

4. Anna, S.L.: Droplets and bubbles in microfluidic devices. Annual Review of Fluid Mechanics 48, 285-309 (2016)

5. Aussillous, P., Quéré, D.: Quick deposition of a fluid on the wall of a tube. Physics of Fluids 12(10), 2367-2371 (2000)

6. Baroud, C.N., Gallaire, F., Dangla, R.: Dynamics of microfluidic droplets. Lab on a Chip 10(16), 2032-2045 (2010)

7. Boden, S., dos Santos Rolo, T., Baumbach, T., Hampel, U.: Synchrotron radiation microtomography of Taylor bubbles in capillary two-phase flow. Experiments in Fluids 55(7), 1768 (2014)

8. Boos, W., Thess, A.: Thermocapillary flow in a Hele-Shaw cell. Journal of Fluid Mechanics 352, 305-330 (1997)

9. Bretherton, F.P.: The motion of long bubbles in tubes. Journal of Fluid Mechanics 10(02), 166-188 (1961)

10. Cantat, I.: Liquid meniscus friction on a wet plate: Bubbles, lamellae, and foams. Physics of Fluids 25(3), 031,303 (2013)

11. Chen, J.D.: Measuring the film thickness surrounding a bubble inside a capillary. Journal of Colloid and Interface Science 109(2), 341-349 (1986)

12. Cherukumudi, A., Klaseboer, E., Khan, S.A., Manica, R.: Prediction of the shape and pressure drop of Taylor bubbles in circular tubes. Microfluidics and Nanofluidics 19(5), 1221-1233 (2015)

13. Derjaguin, B.: On the thickness of the liquid film adhering to the walls of a vessel after emptying. Acta Physicochimica URSS 20(43), 349-352 (1943)

14. Eggers, J., Villermaux, E.: Physics of liquid jets. Reports on Progress in Physics 71(3), 036,601 (2008)

15. Fairbrother, F., Stubbs, A.E.: Studies in electro-endosmosis. Part VI. The bubble-tube method of measurement. Journal of the Chemical Society (Resumed) pp. 527-529 (1935)

16. Fuerstman, M.J., Lai, A., Thurlow, M.E., Shevkoplyas, S.S., Stone, H.A., Whitesides, G.M.: The pressure drop along rectangular microchannels containing bubbles. Lab on a Chip 7(11), 1479-1489 (2007)

17. Ganesan, S., Hahn, A., Simon, K., Tobiska, L.: ALE-FEM for Two-Phase and Free Surface Flows with Surfactants. In: Transport Processes at Fluidic Interfaces, pp. 5-31. Springer (2017)

18. Ganesan, S., Tobiska, L.: Arbitrary Lagrangian-Eulerian finiteelement method for computation of two-phase flows with soluble surfactants. Journal of Computational Physics 231(9), 3685-3702 (2012)

19. Giavedoni, M.D., Saita, F.A.: The axisymmetric and plane cases of a gas phase steadily displacing a Newtonian liquid - A simultaneous solution of the governing equations. Physics of Fluids 9(8), 2420-2428 (1997)

20. Giavedoni, M.D., Saita, F.A.: The rear meniscus of a long bubble steadily displacing a Newtonian liquid in a capillary tube. Physics of Fluids 11(4), 786-794 (1999)

21. Günther, A., Jensen, K.F.: Multiphase microfluidics: from flow characteristics to chemical and materials synthesis. Lab on a Chip 6(12), 1487-1503 (2006)

22. Gupta, R., Leung, S.S.Y., Manica, R., Fletcher, D.F., Haynes, B.S.: Three Dimensional Effects in Taylor Flow in Circular Microchannels. La Houille Blanche (2), 60-67 (2013)

23. Hadikhani, P., Hashemi, S.M.H., Balestra, G., Zhu, L., Modestino, M.A., Gallaire, F., Psaltis, D.: Inertial manipulation of bubbles in rectangular microfluidic channels. Lab on a Chip 18(7), 10351046 (2018)
24. Han, Y., Shikazono, N.: Measurement of liquid film thickness in micro square channel. International Journal of Multiphase Flow 35(10), 896-903 (2009)

25. Hazel, A.L., Heil, M., Waters, S.L., Oliver, J.M.: On the liquid lining in fluid-conveying curved tubes. Journal of Fluid Mechanics 705, 213-233 (2012)

26. Heil, M.: Finite Reynolds number effects in the Bretherton problem. Physics of Fluids 13(9), 2517-2521 (2001)

27. Herrada, M.A., Ganan-Calvo, A.M., Guillot, P.: Spatiotemporal instability of a confined capillary jet. Physical Review E 78(4), 046,312 (2008)

28. Hodges, S.R., Jensen, O.E., Rallison, J.M.: The motion of a viscous drop through a cylindrical tube. Journal of Fluid Mechanics 501, 279-301 (2004)

29. Huerre, A., Theodoly, O., Leshansky, A.M., Valignat, M.P., Cantat, I., Jullien, M.C.: Droplets in microchannels: dynamical properties of the lubrication film. Physical Review Letters 115(6), 064,501 (2015)

30. Jakiela, S., Makulska, S., Korczyk, P.M., Garstecki, P.: Speed of flow of individual droplets in microfluidic channels as a function of the capillary number, volume of droplets and contrast of viscosities. Lab on a Chip 11(21), 3603-3608 (2011)

31. Khodaparast, S., Kim, M.K., Silpe, J.E., Stone, H.A.: Bubbledriven detachment of bacteria from confined microgeometries. Environmental Science \& Technology 51(3), 1340-1347 (2017)

32. Khodaparast, S., Magnini, M., Borhani, N., Thome, J.R.: Dynamics of isolated confined air bubbles in liquid flows through circular microchannels: an experimental and numerical study. Microfluidics and Nanofluidics 19(1), 209-234 (2015)

33. Klaseboer, E., Gupta, R., Manica, R.: An extended Bretherton model for long Taylor bubbles at moderate capillary numbers. Physics of Fluids 26(3), 032,107 (2014)

34. Köhler, J., Cahill, B.: Micro-Segmented Flow: Applications in Chemistry and Biology. Biological and Medical Physics, Biomedical Engineering. Springer Berlin Heidelberg (2014)

35. Kreutzer, M.T., Kapteijn, F., Moulijn, J.A., Kleijn, C.R., Heiszwolf, J.J.: Inertial and interfacial effects on pressure drop of Taylor flow in capillaries. AIChE Journal 51(9), 2428-2440 (2005)

36. Lac, E., Sherwood, J.D.: Motion of a drop along the centreline of a capillary in a pressure-driven flow. Journal of Fluid Mechanics 640, 27-54 (2009)

37. Ładosz, A., Rigger, E., von Rohr, P.R.: Pressure drop of threephase liquid-liquid-gas slug flow in round microchannels. Microfluidics and Nanofluidics 20(3), 49 (2016)

38. Landau, L., Levich, B.: Dragging of a Liquid by a Moving Plate. Acta Physicochimica URSS 17(42), 42-54 (1942)

39. Langewisch, D.R., Buongiorno, J.: Prediction of film thickness, bubble velocity, and pressure drop for capillary slug flow using a CFD-generated database. International Journal of Heat and Fluid Flow 54, 250-257 (2015)

40. Leung, S.S.Y., Gupta, R., Fletcher, D.F., Haynes, B.S.: Effect of flow characteristics on Taylor flow heat transfer. Industrial \& Engineering Chemistry Research 51(4), 2010-2020 (2011)

41. Li, J.: Macroscopic Model for Head-On Binary Droplet Collisions in a Gaseous Medium. Physical Review Letters 117(21), 214,502 (2016)

42. Magnini, M., Ferrari, A., Thome, J.R., Stone, H.A.: Undulations on the surface of elongated bubbles in confined gas-liquid flows. Physical Review Fluids 2(8), 084,001 (2017)

43. Martinez, M.J., Udell, K.S.: Axisymmetric creeping motion of drops through circular tubes. Journal of Fluid Mechanics 210, 565-591 (1990)

44. Mikaelian, D., Haut, B., Scheid, B.: Bubbly flow and gas-liquid mass transfer in square and circular microchannels for stressfree and rigid interfaces: dissolution model. Microfluidics and Nanofluidics 19(4), 899-911 (2015) 
45. Nagel, M., Gallaire, F.: Boundary elements method for microfluidic two-phase flows in shallow channels. Computers \& Fluids 107, 272-284 (2015)

46. Park, C.W., Homsy, G.M.: Two-phase displacement in Hele-Shaw cells: theory. Journal of Fluid Mechanics 139, 291-308 (1984)

47. Ratulowski, J., Chang, H.C.: Transport of gas bubbles in capillaries. Physics of Fluids A: Fluid Dynamics (1989-1993) 1(10), 1642-1655 (1989)

48. Reinelt, D.A., Saffman, P.G.: The penetration of a finger into a viscous fluid in a channel and tube. SIAM Journal on Scientific and Statistical Computing 6(3), 542-561 (1985)

49. Rivero-Rodriguez, J., Scheid, B.: Bubble dynamics in microchannels: inertial and capillary migration forces. Journal of Fluid Mechanics 842, 215-247 (2018)

50. de Ryck, A.: The effect of weak inertia on the emptying of a tube. Physics of Fluids 14(7), 2102-2108 (2002)

51. Saffman, P.G., Taylor, G.: The penetration of a fluid into a porous medium or Hele-Shaw cell containing a more viscous liquid. Proceedings of the Royal Society of London. Series A. Mathematical and Physical Sciences 245(1242), 312-329 (1958)

52. Schwartz, L.W., Princen, H.M., Kiss, A.D.: On the motion of bubbles in capillary tubes. Journal of Fluid Mechanics 172, 259-275 (1986)

53. Shen, E.I., Udell, K.S.: A finite element study of low Reynolds number two-phase flow in cylindrical tubes. Journal of Applied Mechanics 52(2), 253-256 (1985)

54. Stone, H.A.: Interfaces: in fluid mechanics and across disciplines. Journal of Fluid Mechanics 645, 1-25 (2010)

55. Stone, H.A., Stroock, A.D., Ajdari, A.: Engineering flows in small devices: microfluidics toward a lab-on-a-chip. Annual Review of Fluid Mechanics 36, 381-411 (2004)

56. Taylor, G.I.: Deposition of a viscous fluid on the wall of a tube. Journal of Fluid Mechanics 10(02), 161-165 (1961)

57. Tsai, T.M., Miksis, M.J.: Dynamics of a drop in a constricted capillary tube. Journal of Fluid Mechanics 274, 197-217 (1994)

58. Warnier, M.J.F., De Croon, M.M., Rebrov, E.V., Schouten, J.C.: Pressure drop of gas-liquid Taylor flow in round micro-capillaries for low to intermediate Reynolds numbers. Microfluidics and Nanofluidics 8(1), 33 (2010)

59. Wong, H., Radke, C.J., Morris, S.: The motion of long bubbles in polygonal capillaries. Part 1. Thin films. Journal of Fluid Mechanics 292, 71-94 (1995)

60. Wong, H., Radke, C.J., Morris, S.: The motion of long bubbles in polygonal capillaries. Part 2. Drag, fluid pressure and fluid flow. Journal of Fluid Mechanics 292, 95-110 (1995)

61. Zhu, L., Gallaire, F.: A pancake droplet translating in a Hele-Shaw cell: lubrication film and flow field. Journal of Fluid Mechanics 798, 955-969 (2016) 